Article

\title{
Does Reference Dependence Impact Intervention Mechanisms in Vaccine Markets?
}

\author{
Feiyu Guo ${ }^{1,2}$ and Erbao Cao 1,2,* \\ 1 College of Economics and Trade, Hunan University, Changsha 410079, China; guofeiyu@hnu.edu.cn \\ 2 Hunan Province Key Laboratory of Logistics Information and Simulation Technology, \\ Changsha 410079, China \\ * Correspondence: caoerbao@hnu.edu.cn
}

Received: 13 July 2020; Accepted: 5 August 2020; Published: 7 August 2020

\begin{abstract}
Prior research suggests that, to maintain sustainable health, inefficient vaccine markets need to be intervened by government subsidy. However, the effectiveness of these intervention mechanisms is often reduced by the absence of reference dependence preference. Our paper introduces this preference as the psychological disutility of overproduction and underproduction, and then uses game-theoretic way to find that reference dependence has implications on one-sided (pure demand or supply side) and two-sided intervention mechanisms. (i) The positive impact is that this preference helps both pure demand-sided and specific two-sided intervention mechanisms not only to maximize social welfare, but also to achieve diverse targets: pure demand-sided one reduces government interventions while specific two-sided ones achieves budget neutrality. (ii) The negative impact is that, although maximizing social welfare, reference dependence makes general two-sided intervention mechanisms complex. This complexity is reflected in differences in intervention's extent (i.e., a change in payment extent used to address inefficient vaccine markets) and structure (i.e., an adjustment from subsidy alone to subsidy/tax/zero schemes). These finds guide governments with diverse targets to design the corresponding intervention mechanisms to maintain sustainable health.
\end{abstract}

Keywords: behavioral operations; reference dependence; intervention mechanisms; vaccine market; game theory; subsidy and tax

\section{Introduction}

The United Nations Sustainable Development Goals (including 17 goals) adopted by world leaders in September 2015 set out a vision for a world free of poverty, hunger, disease and want by the year 2030. In these 17 goals, health has a central place in the third goal: 'Ensure healthy lives and promoting well-being for all at all ages'. Additionally, almost all of the other 16 goals are directly related to health or will contribute to health indirectly [1].

In reality, infectious diseases, such as influenza, the SARS, Ebola viruses and COVID-19, often threat individuals' health and safety. To mitigate these threats, enhancing vaccine coverage is generally employed as a cost-effective prevention method [1]. Unfortunately, a traditional vaccine market may be inefficient due to vaccination externality or yield uncertainty that are significant features in vaccine markets (more detailed reasons can be found in Section 2.1). To address such inefficiency, some papers have proposed one- or two-sided intervention mechanisms where all decision makers are assumed to be perfectly rational or profit-maximizing, e.g., [2-4].

These assumptions, however, are challenged if we tend to fully understand their decision processes and associated objectives by recent empirical findings or realistic cases. In empirical research, decision makers appear to differ in arriving at profit-maximization decisions prescribed by standard economy theory within an uncertain environment, e.g., $[5,6]$. Indeed, reference dependence is one of the most 
consistent and widespread non-standard preferences, e.g., [5]. That is, the outcome of a choice assessed by a decision maker depends not only on its intrinsic utility but also on psychological utility [7]. Through experimental economics, this psychological utility is often characterized by the costs from leftovers and stockouts relative to fixed order quantity in the environment of demand uncertainty [8]. Borrowing from this characterization, our paper considers that, in the vaccine market, reference dependence can be derived from yield uncertainty. This is because, relative to vaccine demand from fixed pre-order, eventual vaccine quantity is significantly uncertain [3], and such uncertainty entices a vaccine manufacturer to experience psychological disutility of overproduction and underproduction. Our paper characterizes this psychological disutility as reference dependence preference and then introduces this preference into a manufacturer's objective.

Importantly, this introduction in a manufacturer's objective meets the requirements of realistic cases, especially in the setting where a vaccine saves lives and is beyond a normal commodity. One case is from Sanofi and Johnson \& Johnson, both of which are primary pharmaceutical manufacturers. With no profit motives, they have claimed to expand vaccine supply on a large scale and will sell these vaccines after the success of developing a COVID-19 vaccine [6]. This case indicates that the objective of these manufacturers has experienced the transition from profits maximization, e.g., [2,4], to utilities maximization (i.e., no profit motive). Another case is from GlaxoSmithKline (hereafter, GSK), a primary vaccine manufacturer. In response to the 2016 humanitarian crisis in Syria and South Sudan, GSK pledged to expend capacity and to sell essential vaccines at the lowest price to refugees [9]. This case implies that GSK puts emphasis on consumer surplus associated with the availability and affordability of a vaccine so as to achieve social or individuals' healthy development. In fact, this emphasis served as a significant component of corporate social responsibility and can be reflected in the psychological disutility of underproduction in our model.

After showing the reasons of introducing this preference, one question naturally arises: Does the presence of reference dependence affect the design of intervention mechanisms? In other words, when considering a manufacturer's reference dependence, which intervention mechanisms should be favored by governments? To answer this question, we build a three-stage model that captures the interactions between a government, a manufacturer and individuals. In the first stage, the government designs one- or two-sided intervention mechanisms to maximize social welfare. In the second stage, given these intervention mechanisms, a reference-dependent manufacturer who maximizes the expected utility determines vaccine prices and production quantities, simultaneously. In the third stage, rational individuals make their vaccination decisions based on these intervention mechanisms and manufacturer's decisions. Our equilibrium analysis of this model finds that the different level of reference dependence preference categorizes all intervention mechanisms into one-sided (including pure demand and pure supply ones) and two-sided (including specific and general ones). More importantly, the implications of this preference in these intervention mechanisms are different (Table 1).

Table 1. The impact of reference dependence in intervention mechanisms.

\begin{tabular}{cccc}
\hline \multirow{2}{*}{ Intervention Types } & \multicolumn{2}{c}{ Targets Achieved by Different Intervention Mechanisms } \\
\cline { 2 - 4 } & $\begin{array}{c}\text { Maximizing Social } \\
\text { Welfare }\end{array}$ & The Positive Impact & The Negative Impact \\
\hline $\begin{array}{c}\text { Pure demand-sided } \\
\text { mechanisms } \\
\begin{array}{c}\text { Pure supply-sided } \\
\text { mechanisms }\end{array}\end{array}$ & Yes & $\begin{array}{c}\text { Reduce government } \\
\text { interventions }\end{array}$ & None \\
$\begin{array}{c}\text { Specific two-sided } \\
\text { mechanisms } \\
\begin{array}{c}\text { General two-sided } \\
\text { mechanisms }\end{array}\end{array}$ & Yo & None & None \\
neutrality & None & None \\
\hline $\begin{array}{c}\text { Note: 'None' represents that reference dependence does not have positive or negative impact in intervention } \\
\text { mechanisms. }\end{array}$
\end{tabular}


First, the positive impact is that, under certain conditions, reference dependence helps some intervention mechanisms (i.e., pure demand-sided and specific two-sided) not only to maximize social welfare, but also to achieve diverse goals. These goals are reflected in the ease of implementation, such as reducing government interventions or achieving budget neutrality. On one hand, pure demand-sided intervention mechanisms reduce government interventions in supply side. The underlying reason is that, under certain conditions, a reference-dependent manufacturer (e.g., social entrepreneur) places an emphasis on the psychological disutility of vaccine shortages (or underproduction) and thus adjusts the corresponding production quantity to the social optimal level. On the other hand, specific two-sided intervention mechanisms can achieve budget neutrality by transfer payments (i.e., subsidies or taxes) made between vaccinated individuals and a reference-dependent manufacturer. Additionally, if pure demand-sided intervention mechanisms are modified by transfer payments made between vaccinated and unvaccinated individuals, this modified one can also achieve budget neutrality. Thus, both modified demand-sided and special two-sided intervention mechanisms are always equivalent in maximizing social welfare, even maintaining budget-neutral in expectation. This desired result provides a flexibility for the government to design intervention mechanisms.

Second, the negative impact is that, when maximizing social welfare, the presence of this preference makes general two-sided intervention mechanisms complex (pure supply-sided intervention mechanisms fail to maximize social welfare due to vaccine externality). This complexity focuses on differences in intervention's extent and structure. On one hand, intervention's extent represents the optimal payment extent used for intervention mechanisms (or rather, the size of tax or subsidy). Its difference implies a change in the extent of the optimal payment caused by reference dependence preference. Ignoring this preference and the resulting change often lead to over- (or under-) use tax and subsidy in both sides (supply and demand). On the other hand, intervention's structure means that the direction of the optimal payment used to address the inefficient vaccine market may be positive (subsidy), zero, or negative (tax). Its difference implies that, under general two-sided intervention mechanisms, the presence of reference dependence leads to an adjustment in the intervention's structure from subsidy alone to subsidy/tax/zero schemes. Ignoring this preference and the resulting adjustment often leads to misusing subsidy in both supply and demand sides. Therefore, the differences in intervention's extent and structure should be stressed during the design of general two-sided intervention mechanisms.

\section{Literature Review}

\subsection{Yield Uncertainty and Vaccination Externality}

Inefficiency in the vaccine market is primarily caused by yield uncertainty (supply side) or vaccine externality (demand side), both of which will be illustrated in turn.

First, yield uncertainty is often the major reason of supply shortages. When considering a vaccine manufacturer in a complete information, Chick, Mamani and Simchi-Levi [2] first show that both yield uncertainty and the resulting production risk are taken by a vaccine manufacturer alone, leading to vaccine shortages. After that, Deo and Corbett [10] and Chick et al. [11] further conduct research in the context of yield uncertainty: the former extends a vaccine manufacturer to multiple manufacturers in Cournot competition while the latter extends complete information to incomplete ones. Aside from yield uncertainty, other causes of supply shortages include inefficient allocation mechanisms [12] and multiple sources of uncertainty [13].

Second, suboptimal demand for vaccines is generally attributed to vaccination externality, e.g., [14]. Such externality means that, as the fraction of vaccinated individuals increases, infection probability declines and then these unvaccinated individuals tend to reject vaccination. In the above works, vaccination externality is served as an economic instrument for unvaccinated individuals that can protect themselves from infection. In addition to economic issues, social and behavioral issues also influence vaccination coverage, such as altruism [15], anticipated regret and past behaviors [16], reference 
point formation and updating [17] as well as contextual, individual and group, and vaccine-specific influences [18].

In diverging from these conclusions mentioned above, our model shows that vaccine characteristics and the resulting behavioral issues jointly lead to the inefficiency in the vaccine market. Specifically, reference dependence, yield uncertainty and vaccination externality together give rise to a reduction in both production quantity and vaccination coverage.

\subsection{Intervention Mechanism through Subsidy and Tax}

Subsidy and tax are extensively used to coordinate the conflicting incentives of players, thus achieving socially optimal levels in many areas, such as vaccines, green technology and carbon emission [3,19-21]. This subsection will review the literature regarding the design of intervention mechanisms (i.e., subsidy and tax) from the perspective of one and two sides.

First, one-sided intervention mechanisms include pure supply or demand side. On one hand, pure supply-sided mechanisms focus on addressing production issues under the conditions of fixed or exogenous demand. Chick, Mamani and Simchi-Levi [2] use cost-sharing contracts to reduce production costs of a vaccine manufacturer. Levi et al. [22] show that allocating uniform subsidy to firms with different efficiency levels can increase market consumption. Both buy-back-and-late-rebate contract [13] and the optimal menu of output-based contracts [11] can address the question of the tradeoff between vaccine quantity and on-time delivery. On the other hand, pure demand-sided mechanisms are composed of two types: allocation and economic mechanisms. The first type generally involves prioritization [23] and compulsory vaccination [24]. The second type includes subsidy/tax schemes, which are designed as single-period, e.g., [4] and two-periods, e.g., [25].

Second, two-sided intervention mechanisms include two parts: sales subsidy (or sales rebates) on the demand side and cost subsidy on the supply side. Generally, the two parts are acted as substitutes or complements in achieving a targeted level when considering different settings. In the case of substitutes, they are often comparable [26]. In the case of complements, Adida, Dey and Mamani [3] reveal that two-sided mechanisms only can achieve optimal levels of production quantity and vaccine coverage simultaneously.

\subsection{Behavioral Issues and Intervention Mechanisms}

Behavioral operations constitute a well-received research domain [27]. Below, we review works most closely related to two aspects: (i) whether behavioral issues can address inefficient markets or not and (ii) (if any) which intervention types should be designed when considering these behavioral issues.

In the first aspect, economic interventions including subsidy/tax schemes serve as major instruments to address the inefficiency of a decentralized system caused by diverse incentives of players, such as buy-back-revenue-sharing contract [28] and a composite contract (combining buy-back, and sales rebate and penalty contracts in Giri et al. [29]). Under certain conditions, however, behavioral issues can do so, such as fairness concerns [30] and overconfidence [31]. In the second aspect, behavioral issues should be considered when designing diverse intervention mechanisms, such as peer-induced and distributional fairness concern [32], reference points [33,34] and loss aversion [35].

Rather than focusing on economic issues alone, our paper introduces reference-dependent preferences underlying behavioral issues into the design of intervention mechanisms. More importantly, reference dependence has the positive and negative impacts in intervention mechanisms.

\section{Model}

We consider that, before the process of manufacturing vaccines, a government offers intervention mechanisms to manage vaccine markets where a reference-dependent manufacturer offers a certain type of vaccine to a population of individuals. To characterize the strategic interactions between a government, a manufacturer and individuals, our paper builds a three-stage sequential decision-making model (see Figure 1). In the first stage, the government maximizes social welfare and maintains 
individuals' health by choosing one- (pure supply or demand) or two-sided intervention mechanisms. These intervention mechanisms include two components: One is with respect to demand side, i.e., offering a purchase subsidy/tax (indexed by s) to rational individuals, affects vaccine price and then vaccination coverage; the other is with respect to the supply side, i.e., offering a cost subsidy/tax (indexed by $e$ ) to a vaccine manufacturer affects production quantity. In the second stage, considering these intervention mechanisms as given and anticipating individuals' responses, a reference-dependent manufacturer maximizing expected utility (such as Sanofi) simultaneously determines production quantity and vaccine price. Third, rational individuals determine whether to get vaccinated or not depending on manufacturer's decisions and government's intervention mechanisms. In what follows, we will use backward induction to characterize the subgame equilibrium of this three-stage decision-making model. All notational symbols and their meanings will be listed in Appendix C.

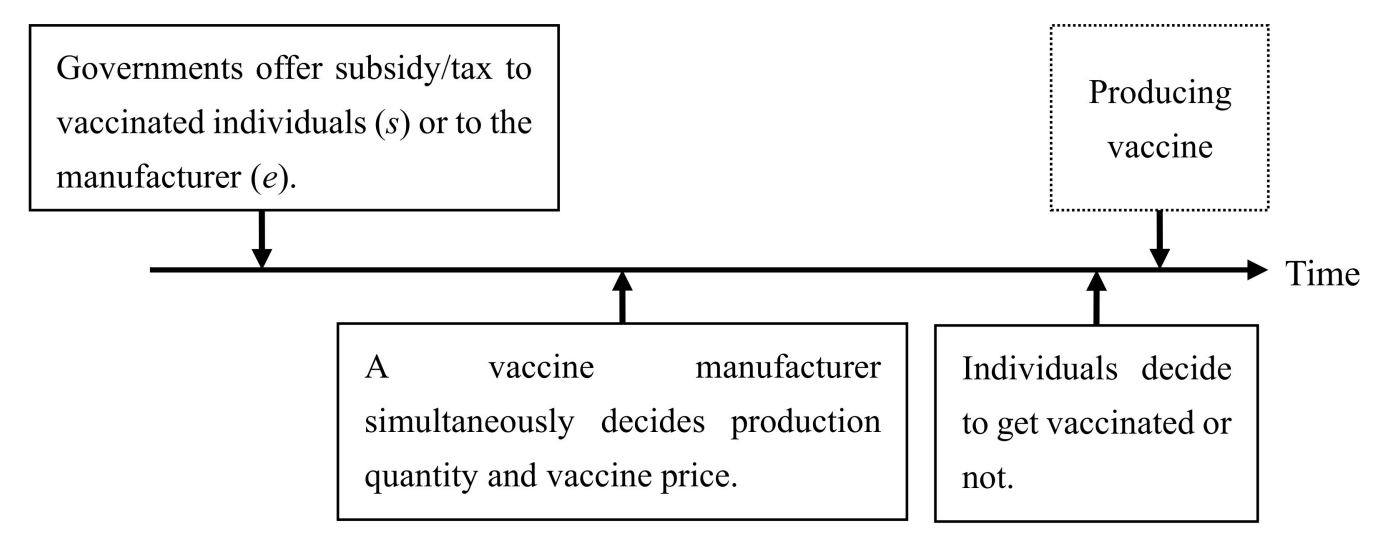

Figure 1. The sequence of events.

\subsection{Demand Model}

Borrowing from existing literatures [3], we first describe the dynamic infection probability, a distinguishing feature of vaccines, and then characterize individuals' behaviors and their resulting vaccine demand.

\subsubsection{Infection Probability}

The probability of infection varies due to specifics of both epidemic models and different groups. These specifics will be analyzed in turn.

First, we consider a standard susceptible-infected-recovered (SIR) in Mamani et al. [36] where $r(f)$ refers to the infection probability for the entire population as a function of the vaccinated fraction (denoted as $f$ ) and satisfies:

$$
r(f)= \begin{cases}0, & \text { if } f>F=\frac{R_{0}-1}{\phi R_{0}}, \\ 1-\phi f-\frac{1}{R_{0}}, & \text { otherwise, }\end{cases}
$$

From this equation, the infection probability for the entire population depends on the interactions between basic reproduction number $\left(R_{0}\right)$ and vaccine efficacy $(\phi) . R_{0}$ serves as an indicator of the infectiousness of a disease: when $R_{0} \leq 1$, an infected individual does not spread the epidemic disease to others and a disease breakout will not occur; by contrast, when $R_{0}>1$, such an infected individual can cause other individuals to be infected and thereby spur disease transmission. To reduce the probability of disease transmission, vaccines are used as preventative tools and their effectiveness (denoted as $\phi$ ) is a major indicator to measure the probability of vaccinated individuals not becoming infected. The greater $\phi$ is, the less probable a vaccinated individual is to become infected. $\phi=1$ captures a perfect vaccine. 
By combining $\phi$ and $R_{0}$, we have the critical vaccination fraction $F=\frac{R_{0}-1}{\phi R_{0}}$ and this fraction captures the lowest level of vaccinated coverage to fully eliminate disease transmission. When $f>F$, herd immunity occurs and unvaccinated individuals are protected from infection. This case can be considered as a significant feature of vaccination externality. To eliminate the uninteresting case, we denote $\bar{F}=\min \{F, 1\}$ to limit the range of $\mathrm{F}$ and the case of $R_{0}>1$ will be considered only in the remainder of this paper.

Second, we consider that the infection probability also depends on the different groups involved (including unvaccinated and vaccinated groups). We denote $P(f)$ and $p(f)$ as the infection probability of unvaccinated and vaccinated fractions, respectively. From works given in Mamani, Adida and Dey [36], we have:

$$
\begin{gathered}
p(f)=\eta(1-\phi) r(f), \text { and } \\
P f=\frac{r(f)-f p(f)}{1-f},
\end{gathered}
$$

where is constant that can be adjusted to satisfy the approximation of $p(f)$. Note that implies the feature of vaccination externality. That is, the infection probability of the unvaccinated fraction diminishes as the fraction of vaccinated individuals increases when $f \leq F$. This diverges from the role of herd immunity that disease transmission is eliminated and thus the infection probability is zero when $f>F$.

\subsubsection{Individuals' Decisions}

In our paper, a continuum of heterogeneous individuals are characterized by the relative direct and indirect costs of infection (indexed by $u$ ), such as outpatient visits and hospitalization [23]. The relative value in our paper can be transformed into the corresponding absolute values through multiplying by $L$ (this way is also applied in other relative values in the rest of our paper). $u$ is assumed to be uniformly distributed over an interval [0,1]. These individuals have two opinions to reduce losses from infection disease. When an individual with $u$ exhibits a greater willingness to get vaccinated, he/she will pay the amount of both vaccine price (indexed by ) and demand-sided subsidy/tax $s$, but may still become infected with the probability $p(f)$ due to vaccine efficacy. This yields the expected cost, $w-s+u p(f)$. Alternatively, when such an individual tends to refuse vaccination, he/she suffers from a higher level of infection probability $P(f)$ and yields the expected cost $u P(f)$. As a result, for a marginal individual (denoted as $\bar{u}$ ) who is indifferent in refusing or receiving vaccination, we have $w-s+\bar{u} p(f)=\bar{u} P(f)$. If $f=1-\bar{u}$, we have $w=(1-f)[P(f)-p(f)]+s$. After defining $T(f)=\theta^{r}(f)=[1-\eta(1-\phi)[r(f)$ from Mamani, Adida and Dey [36], we get the following relationship between vaccine prices and vaccine coverage:

$$
w=(1-f)[P(f)-p(f)]+s=\theta r(f)+s=T(f)+s
$$

Equation (1) indicates that individuals make their vaccination decisions in such a way that balances between vaccine price and demand-sided subsidy/tax, $w$-s, and a marginal reduction in infection probability, $r(f)-p(f)$.

\subsection{Supply Model}

This subsection will first describe two components: yield uncertainty and reference dependence. Subsequently, the two components will be incorporated into the expected utility of the manufacturer and thereby, the optimal decisions in the supply side are obtained.

\subsubsection{Yield Uncertainty and Reference Dependence}

Considerable uncertainty in the obtained vaccines are a major characteristic of the production process of influenza vaccine [2]. We assume that stochastically proportional yield indexed by $u_{E}$ describes the uncertainty in obtained vaccine quantity [3]. It has a mean value of $E\left[u_{E}\right]=u$ with a probability density of $j(\cdot)$, a distribution function of $J(\cdot)$ and a complementary distribution of $\bar{J}(\cdot)$ for 
an interval of $[0, \bar{U}]$. Further, if $q$ is denoted as target production quantity, the obtained number of vaccines is $q_{v}=u_{E} q$.

In the setting of yield uncertainty, we argue that a vaccine manufacturer often experiences psychological disutility of overproduction or underproduction (see Section 1). This disutility is termed as implicitly reference-dependent preference, which will serve as endogenously influencing factors in evaluating the effectiveness of his/her decisions. Borrowed from the way of modeling reference dependence [8], our paper characterizes this preference as the following psychological disutility in the setting of yield uncertainty:

$$
U_{R}=\alpha \cdot \max \left(f-u_{E} q, 0\right)+\beta \cdot \max \left(u_{E} q-f, 0\right) .
$$

In this equation, parameter $\alpha$ (or $\beta$ ) is nonnegative and measures the psychological per-unit disutility of underproduction (or overproduction). When $u_{E} q=f$, the obtained vaccine quantity is equal to the level of vaccination coverage and the psychological disutility resulting from reference dependence is zero. When $u_{E} q<f$, underproduction occurs and then a manufacturer exhibits the corresponding psychological disutility $\alpha\left(f-u_{E} q\right)$. Similarly, when $u_{E} q>f$, overproduction occurs and then the manufacturer exhibits the corresponding psychological disutility $\beta\left(u_{E} q-f\right)$.

\subsubsection{Manufacturer's Decisions}

In the second stage, considering intervention mechanisms as given, a reference-dependent manufacturer specifies vaccine price $(w)$ and production quantity $(q)$ simultaneously, while incurring production $\operatorname{cost}(c)$ and incentive scheme $(e<c)$ per unit. As a function of vaccine price and production quantity, the vaccine manufacturer's utility is:

$$
U(q, w)=\pi(q, w)+U_{R}(q, w)=w \cdot \min \left\{u_{E} q, f\right\}-(c-e) q+\alpha \cdot \max \left(f-u_{E} q, 0\right)+\beta \cdot \max \left(u_{E} q-f, 0\right)
$$

where $\pi(q, w)=w \cdot \min \left\{u_{E} q, f\right\}$. Obviously, this utility is constructed by two components: profits associated with obtained vaccine quantity and vaccination coverage, $\pi(q, w)$, and the psychological disutility associated with reference dependence, $U_{R}(q, w)$. Since the manufacturer can anticipate individuals' response, we recall Equation (1) and have the expected utility of the manufacturer:

$$
\begin{gathered}
E[U(q, f)]=\underbrace{\int_{0}^{\frac{f}{q}}[(T(f)+s) x q-(c-e) q] d J(x)+\int_{\frac{f}{q}}^{\bar{u}}[(T(f)+s) f-(c-e) q] d J(x)}_{E[\pi(q, f)]} \\
+\underbrace{\alpha \int_{0}^{\frac{f}{q}}(x q-f) d J(x)+\beta \int_{\frac{f}{q}}^{\bar{u}}(f-x q) d J(x)}_{E\left[U_{R}(q, f)\right]} .
\end{gathered}
$$

In this equation, as an increase in production quantity given the fixed level of vaccine demand, the expected psychological disutility of underproduction decreases while the expected psychological disutility of overproduction increases. Thus, to maximize the expected utility, a reference-dependent manufacturer faces a psychological tradeoff between underproduction and overproduction. Based on this expected utility, we make a reasonable assumption for economic meanings:

Assumption 1. The expected utility exceeds the production cost, $(w+\alpha) \mu-c>0$.

This assumption guarantees the optimal production quantity is not zero. That is, only if the expected utility exceeds the production cost, a vaccine manufacturer enters the vaccine market and produces vaccines. Based on this assumption, we take two steps to characterize the decisions of a reference-dependent manufacturer by adopting the method of Adida, Dey and Mamani [3]. First, 
given that $f<\bar{F}$, we derive optimal production quantity indexed by $q_{D}^{*}(f)$. Second, we solve optimal vaccination coverage $f_{D}^{*}$ given the optimal quantity $q_{D}^{*}(f)$. Note that Lemma 1 and Theorem 1 (both of which will be shown later) consider the optimal decisions under the decentralized system (i.e., $s=e=0$ ), which contributes to demonstrating the importance of intervention mechanisms. After showing this importance, we consider how to choose different kinds of intervention mechanisms from the perspective of governments.

Now, the following lemma characterizes the first step.

Lemma 1. $E[U(q, f)]$ is concave for all $q$ values, and a unique optimal quantity $\hat{q}_{D}$ maximizes the expected utility and satisfies the following first-order condition:

$$
[T(f)+\alpha+\beta] \int_{0}^{\frac{f}{q}} x d J(x)=\beta \mu+c
$$

Furthermore, the optimal quantity $\hat{q}_{D}(f)$ is increasing (or decreasing) in $\alpha$ ( or $\beta$ ). Then, $q_{D}^{*}(f)$ can be given by:

$$
q_{D}^{*}(f)= \begin{cases}\hat{q}_{D}(f), & \text { if }[T(f)+\alpha] \mu \geq c \\ 0, & \text { otherwise }\end{cases}
$$

All proofs can be found in Appendix A. Lemma 1 indicates that the optimal production quantity is affected not only by economic issues (i.e., yield uncertainty, production cost and vaccination coverage) but also by behavioral issues (i.e., the psychological cost of reference dependence). More precisely, the bigger level of $\alpha$ (or $\beta$ ) increases (or decreases) the optimal quantity.

Next, to address the problem of the optimal vaccination coverage selected by the referencedependent manufacturer, we can reduce his/her expected utility to a function of a single decision variable $f$ from Lemma 1 . In turn, we have the following theorem.

Theorem 1. From the distribution function $J(z)$ for $z,(\alpha, \beta)$ satisfies the following conditions:

$$
\left[c+\beta \mu-\frac{\alpha \int_{0}^{z} x d J(x)}{\int_{z}^{\bar{u}} d J(x)}\right] \cdot\left\{2 \rho(z) \int_{z}^{\bar{u}} d J(x)+\left[\int_{0}^{z} x d J(x)+z \int_{z}^{\bar{u}} d J(x)\right] \frac{d \rho(z)}{d z}\right\} \geq 0
$$

where $\rho(z)=\frac{j(z)}{\int_{z}^{\bar{u}} d J(x)}$ is the hazard rate for yield uncertainty. Thus, as a unique vaccination coverage for region $\left(0, \bar{F}-\frac{c}{\mu \theta \phi}+\frac{\alpha}{\theta \phi}\right), f_{D}^{*}$ satisfies $\frac{d E\left[U\left(q_{D}^{*}(f), f\right)\right]}{d f}=0$ when $\bar{F}-\frac{c}{\mu \theta \phi}+\frac{\alpha}{\theta \phi} \geq 0$ and $f_{D}^{*}=0$ otherwise. Accordingly, in the decentralized system, the optimal values regarding vaccine price and quantity are $w_{D}^{*}=\operatorname{\theta r}\left(f_{D}^{*}\right)$ and $q_{D}^{*}=q\left(f_{D}^{*}\right)$, respectively.

Theorem 1 implies that under different conditions of yield uncertainty, a reference-dependent manufacturer enters the vaccine market and elicits optimal values in terms of both vaccine price and production quantity. This theorem extends the results of Adida, Dey and Mamani [3] that a profit-driven manufacturer will enter the vaccine market and choose the optimal values only when $2 \rho(z) \int_{z}^{\bar{u}} d J(x)+\left[\int_{0}^{z} x d J(x)+z \int_{z}^{\bar{u}} d J(x)\right] \frac{d \rho(z)}{d z} \geq 0$. In fact, our model shows that the existence of optimal values is affected by sufficient conditions with two features: yield uncertainty, $\left\{2 \rho(z) \int_{z}^{\bar{u}} d J(x)+\left[\int_{0}^{z} x d J(x)+z \int_{z}^{\bar{u}} d J(x)\right] \frac{d \rho(z)}{d z}\right\}$, and reference dependence, $\left[c+\beta \mu-\frac{\alpha \int_{0}^{z} x d J(x)}{\int_{z}^{\bar{u}} d J(x)}\right]$. More specifically, even when the condition of yield uncertainty satisfies $2 \rho(z) \int_{z}^{\frac{J_{z}}{u}} d J(x)+$ 
$\left[\int_{0}^{z} x d J(x)+z \int_{z}^{\bar{U}} d J(x)\right] \frac{d \rho(z)}{d z}$, a reference-dependent manufacturer with $c+\beta \mu-\frac{\alpha \int_{0}^{z} x d J(x)}{\int_{z}^{\bar{u}} d J(x)}<0$ will enter the vaccine market and then choose the optimal values.

\subsection{Intervention Mechanisms}

Anticipating both the manufacturer's and individuals' responses, the government should design intervention mechanisms to maximize the social welfare. To do so, this subsection constructs the function of social welfare in the centralized system and then derives the first best solutions in terms of both vaccine price and quantity. Through comparing the optimal values in decentralized system with the counterpart in the centralized system, intervention mechanisms are naturally obtained.

\subsubsection{The First Best Solution}

A complete account of social welfare in the centralized system covers four elements: manufacturer's surplus, vaccinated and unvaccinated individuals' surplus, and other surplus [3]. These elements will be shown in turns.

Manufacturer's surplus is equal to the expected utility of the vaccine manufacturer in the decentralized system (see Section 3.2.2). Note that, since there are no intervention mechanisms $(s=e=0)$ in the centralized system $[3,37]$, the vaccine price is $T(f)$ and production cost is $c$. Thus, we have:

Manufacturer's surplus $=\sigma_{M}=T(f) g-c q+\alpha \cdot \max \left(f-u_{E} q, 0\right)+\beta \cdot \max \left(u_{E} q-f, 0\right)$.

Vaccinated individuals' surplus depends on the cost gap between two choices: refusing and accepting vaccination. If individuals are vaccinated at the normalized cost $T(f) g$, they will be infected with a lower probability, $p(g)$, and incur the cost, $\int_{1-g}^{1} x p(g) d x+T(f) g$. Alternatively, if these individuals refuse vaccination and the total fraction to get vaccinated is zero, they may be infected with the probability $r(0)$ and incur the cost $\int_{1-g}^{1} x r(0) d x$. Therefore, from the two choices listed above, we have:

$$
\text { Vaccinated individuals' surplus }=\sigma_{V}=\int_{1-g}^{1} x r(0) d x-\left[\int_{1-g}^{1} x p(g) d x+T(f) g\right] .
$$

Unvaccinated individuals' surplus, different from vaccinated individuals' surplus based on their own choices, is determined as remaining individuals' choices due to vaccination externality. When the remaining individuals choose to get vaccinated, each unvaccinated individual with a normalized cost $u \epsilon(0,1-g)$ has an infection probability $P(g)$. However, when these remaining individuals decide to refuse vaccination, each unvaccinated individual has an infection probability $r(0)$. Calculating the cost difference between the two choices yields:

$$
\text { Unvaccinated individuals' surplus }=\sigma_{N}=\int_{0}^{1-g} x(r(0)-P(g)) d x .
$$

Other surplus includes two components: indirect cost to the society and indirect influence to all individuals. The first component represents that infected individuals incur a loss $\lambda$ on society, such as, lost days of work, e.g., [2]. The second component captures that both underproduction and overproduction have indirect (or positive) influences in all individuals (Note that the positive indirect influence describes the behaviors of all individuals, rather than vaccinated or unvaccinated individuals alone. Thus, this influence will be counteracted in individuals' net utility under the decentralized system). Underproduction induces all individuals to reduce the waiting times of delaying decisions due to, such as, vaccine hesitancy, e.g., [18]. Similarly, overproduction suggests that this production has good quality and can enhance vaccine confidence to overcome infectious diseases [38]. Thus, these influences are denoted as $\tau \cdot \max \left(f-\mathfrak{u}_{E} q, 0\right)$ and $\varphi \cdot \max \left(\mathfrak{u}_{E} q-f, 0\right)$, respectively. $\tau($ or $\varphi)$ reflects per-unit benefit 
from underproduction (or overproduction) and the term $\max \left(f-u_{E} q, 0\right)$ (or $\max \left(u_{E} q-f, 0\right)$ ) captures the corresponding extent [39]. Thus, adding up two components, we get:

$$
\text { Other surplus }=\sigma_{s}=-\lambda r(g)+\tau \cdot \max \left(f-u_{E} q, 0\right)+\varphi \cdot \max \left(u_{E} q-f, 0\right) .
$$

In applying the four elements listed above, we have:

$$
\begin{aligned}
\text { Total social welfare } & =\sigma_{M}+\sigma_{V}+\sigma_{N}+\sigma_{s}=\frac{r(0)}{2}-\frac{(1-g) r(g)+g p(g)}{2}-\lambda r(g)-c q \\
& +(\tau-\alpha) \cdot \max \left(f-\mathbf{u}_{E} q, 0\right)+(\varphi-\beta) \cdot \max \left(\mathbf{u}_{E} q-f, 0\right),
\end{aligned}
$$

whereas before, $g=\min \left\{\mathrm{u}_{E} q, f\right\}$ captures the eventual vaccinated fraction of the population. To obtain differences between our intervention mechanisms (to be shown in the next section) and previous mechanisms in Adida, Dey and Mamani [3], we consider the first best solution as a benchmark by assuming $\tau=\alpha$ and $\varphi=\beta$. Additionally, our major results continue to hold if $\tau$ and $\varphi$ are satisfied by some other conditions (which will be found in Appendix B).

By maximizing the total level of social welfare, governments decide both vaccine quantity and price. This process is the same as Theorem 2, given in Adida, Dey and Mamani [3].

Theorem 2. Let $q^{*}$ and $f^{*}$ be the first best solutions when maximizing social welfare. Let $\hat{q}^{*}$ be the solution of

$$
\frac{1}{2} \int_{0}^{\frac{\bar{F}}{q}}[(2 \lambda+1) \phi+T(x q)+x q T \prime(x q)] x d J(x)-c=0 .
$$

Then,

$$
q^{*}=\left\{\begin{array}{ll}
\hat{q}^{*}, & \text { if } c \leq \mu\left(\phi \lambda+\frac{\phi+T(0)}{2}\right), \\
0, & \text { otherwise, }
\end{array} \text { and } f^{*}=\min \left\{\bar{F}, \bar{U} q^{*}\right\} .\right.
$$

\subsubsection{Government Decisions}

As governments maximize social welfare, they naturally are faced with two questions: The first is whether the vaccine market with reference-dependent manufacturer is efficient or not; if not, the second is, which intervention mechanisms should be designed. The following proposition can answer the first question after comparing the optimal levels (i.e., vaccine quantity and price showed in Lemma 1 and Theorem 1, respectively) in the decentralized system with that in the centralized system (Theorem 2):

Proposition 1. In the vaccine market, both the optimal vaccine price and production quantity selected by a reference-dependent manufacturer in the setting of no intervention mechanisms fail to achieve the first best solutions derived from maximizing the social welfare.

Proposition 1 indicates the importance and necessity of intervention mechanisms because a decentralized system is inefficient in terms of both vaccine price and production quantity. Such an inefficiency is majorly caused by the incompatible incentives of players: A vaccine manufacturer focuses on utility maximization whereas the government pursues to maximize social welfare. To support this causal relationship, two cases can be observed during the process of proofing Proposition 1 . In the case of $f^{*}=\bar{F}<\bar{U} q^{*}$, reference dependence leads to the presence of conflicting conditions, especially when a vaccine manufacturer chooses socially optimal values in terms of both production volume and vaccination coverage. In the case of $f^{*}=\bar{U} q^{*}<\bar{F}$, the optimal level of vaccination coverage is always lower in the decentralized system than in the centralized system. The rational is that the other surplus is overlooked during the process of maximizing the expected utility of the manufacturer. 
Due to inefficient vaccine markets shown in Proposition 1, the second question naturally arises and thereby, the following theorem characterizes government's decisions (The intervention mechanism on the demand side must be a function of vaccination coverage as demonstrated by Adida, Dey, and Mamani [3]. Besides, $\mathrm{s}(f)<w$ satisfies economic meanings).

Theorem 3. Suppose that the first best solutions in terms of production quantity and vaccination coverage (i.e., $f^{*}$ and $q^{*}$ ) are given by Equations (3) and (4), respectively. We get supply-and demand-sided intervention mechanisms satisfying

$$
\begin{aligned}
& e^{(\alpha, \beta)}= \begin{cases}c-\left[T\left(f^{*}\right)\right] s\left(f^{*}\right) \int_{0}^{\frac{f^{*}}{q^{*}}} x d J(x)+\psi(\alpha, \beta), & q^{*}>0 \text { and } \\
0, & q^{*}=0\end{cases} \\
& s^{(\alpha, \beta)}(f)=\left\{\begin{array}{l}
\theta f+\alpha N(f), \text { if } 0 \leq f<f^{*}, \\
\theta f^{*}+\alpha N\left(f^{*}\right), \text { if } f^{*} \leq f<\bar{F}, . \\
-\beta, \text { if } \bar{F} \leq f \leq 1,
\end{array}\right.
\end{aligned}
$$

In these intervention mechanisms, $\psi(\alpha, \beta)=\beta \int_{\frac{f^{*}}{q^{*}}}^{\bar{u}} x d J(x)-\alpha \int_{0}^{\frac{f^{*}}{q^{*}}} x d J(x)$ and $N(f)=\frac{J\left(\frac{f}{q^{*}}\right)}{1-J\left(\frac{f}{q^{*}}\right)-\Delta}$. Additionally, $\Delta$ is a constant satisfying $s^{(\alpha, \beta)}(f)>0$ when $0 \leq f \leq \bar{F}$.

Intuitively, Theorem 3 indicates that supply- or demand-intervention mechanisms include two components: economic issues (i.e., production costs, vaccine prices and vaccination demand) and behavioral issues (i.e., reference dependence). Economic issues are measured by $c-$ $\left[T\left(f^{*}\right)+s\left(f^{*}\right)\right] \int_{0}^{\frac{f^{*}}{q^{*}}} x d J(x)$ in demand-sided intervention mechanisms, and by both $\theta f$ and $\theta f^{*}$ in supply-sided intervention mechanisms. Behavioral issues are characterized by $\psi(\alpha, \beta)$ in demand-sided intervention mechanisms, and by $\alpha N(f), \alpha N\left(f^{*}\right)$ and $-\beta$ in supply-sided intervention mechanisms. These two components will contribute to analyzing the impact of reference dependence in intervention mechanisms, which will be shown in Sections 4 and 5 .

\section{The Positive Impact of Reference Dependence}

Based on supply- and demand-sided intervention mechanisms in Theorem 3, we find the positive impact of reference dependence in intervention mechanisms. Section 4.1 shows that, with the help of reference dependence, all intervention mechanisms are reorganized as one- and two-sided, and then their effectiveness is examined in maximizing social welfare. After that, Section 4.2 shows that reference dependence makes some intervention mechanisms achieve diverse goals while maximizing social welfare.

In the rest of this paper, we give some symbols to facilitate further analysis and their detailed meaning will be shown later. In particular, one-sided intervention mechanisms include pure supply-sided $M^{S}=\left(0, e^{(\alpha, \beta)}\right)$ and pure demand-sided $M^{D}=\left(s^{(\alpha, \beta)}(f), 0\right)$ where $\psi(\alpha, \beta)=e^{(0,0)}$. Two-sided intervention mechanisms $M^{D S}=\left(s^{(\alpha, \beta)}(f), e^{(\alpha, \beta)}\right)$ include a special two-sided one when $B^{T M}(\alpha, \beta)=0$ and a general two-sided one when $B^{T M}(\alpha, \beta) \neq 0$.

\subsection{Examining the Effectiveness of Intervention Mechanisms}

The following proposition examines the effectiveness of one- or two-sided intervention mechanisms. 
Proposition 2. If intervention mechanisms satisfy Equalities (5) and (6), we have:

(i) When $\psi(\alpha, \beta)=-e^{(0,0)}, M^{D}=\left(s^{(\alpha, \beta)}(f), 0\right)$ represents pure demand-sided intervention mechanisms and can induce a reference-dependent manufacturer to choose the first best solution;

(ii) When $\psi(\alpha, \beta) \neq-e^{(0,0)}, M^{D S}=\left(s^{(\alpha, \beta)}(f), e^{(\alpha, \beta)}\right)$ captures two-sided intervention mechanisms and can also do so.

(iii) However, $M^{S}=\left(0, e^{(\alpha, \beta)}\right)$ represents pure supply-sided intervention mechanisms and fails to achieve the first best solution.

Proposition 2 illustrates the effectiveness of intervention mechanisms. First, $M^{S}=\left(0, e^{(\alpha, \beta)}\right)$ fail to maximize the social welfare. Although such mechanisms encourage a vaccine manufacturer to supply the optimal quantity through cost subsidies, vaccination externality as well as the resulting insufficient incentives still occur due to the lack of demand-sided interventions. Consequently, not all vaccines supplied are purchased and the first best solution in vaccination coverage is not achieved. Our outcome differs from the work of Chick, Mamani and Simchi-Levi [2] in that vaccination demand in our model is the result of individuals' decisions rather than being exogenously given by a government. Additionally, our effectiveness analysis regarding $M^{S}=\left(0, e^{(\alpha, \beta)}\right)$ indirectly indicates that demand-sided intervention mechanisms are indispensable to achieving the first best solution of vaccination coverage, especially when individuals themselves make vaccination decisions voluntarily.

Second, $M^{D}=\left(s^{(\alpha, \beta)}(f), 0\right)$ can achieve the first best solutions when a vaccine manufacturer exhibits reference-dependent preference satisfying $\psi(\alpha, \beta)=-e^{(0,0)}$. Since $s^{(\alpha, \beta)}(f)$ can be either positive or negative, the underlying reasons supporting these results are shown as follows. (i) When $0 \leq f<\bar{F}$, the positive $s^{(\alpha, \beta)}(f)$ implies that subsidies are offered to vaccinated individuals. These subsidies increase the willingness to purchase vaccines and stimulate vaccine demand. This expansion of demand entices the vaccine manufacturer to enhance vaccine quantity. In addition to economic forces, vaccine attributes and reference-dependent preference are significantly behavioral forces. In particular, vaccines are life-saving drugs related to prevalent infections and are usually considered humanitarian goods. Under this attribute, implicit reference dependence (satisfying $\left.\psi(\alpha, \beta)=-e^{(0,0)}\right)$ can substitute explicit economic intervention, such as subsidies or taxes in supply side) to achieve the first best solution (see the case of GSK in Section 1). (ii) When $\bar{F} \leq f<1$, the negative $s^{(\alpha, \beta)}(f)$ means that taxes are imposed on vaccinated individuals. This intervention mechanism shirks vaccine demand but increases the possibility of overproduction. Thus, the manufacturer voluntarily adjusts production quantity to the first best solution.

Third, $M^{D S}=\left(s^{(\alpha, \beta)}(f), e^{(\alpha, \beta)}\right)$ can achieve the first best solutions when a vaccine manufacturer exhibits reference-dependent preferences satisfying $\psi(\alpha, \beta) \neq-e^{(0,0)}$. For example, on November 3 , 2014, Dr. Margaret Chan, Director General of the WHO, asserted that manufacturers did not invest in Ebola vaccines for markets that could not pay, leading to lack of vaccines and to enormous losses for families and communities [40]. Thus, governments and the WHO need to adopt two-sided intervention mechanisms: Funding vaccine manufacturers to supply vaccines and subsidizing individuals to get vaccinated.

In conclusion, $M^{S}=\left(0, e^{(\alpha, \beta)}\right)$ fails to achieve the first best solution, whereas both $M^{D}=$ $\left(s^{(\alpha, \beta)}(f), 0\right)$ and $M^{D S}=\left(s^{(\alpha, \beta)}(f), e^{(\alpha, \beta)}\right)$ can do so. In the remaining subsection, we focus on the latter two mechanisms.

\subsection{Making Some Intervention Mechanisms Achieve Diverse Goals While Maximizing Social Welfare}

After examining the effectiveness of intervention mechanisms, we find that reference dependence makes some intervention mechanisms not only maximize social welfare, but also achieve diverse goals. The first goal means that a government intends to design one-sided intervention mechanism instead of two-sided intervention mechanism, if both mechanisms can address an inefficient vaccine market and maximize the social welfare. This is because the former only alters the decisions of 
one side, whereas the latter needs to change the decisions of both sides. The second goal denotes that all payments (including subsidies and taxes) used to coordinate the vaccine market add up to zero. Therefore, reference dependence yields positive impacts if selecting certain conditions about psychological disutility makes some intervention mechanisms achieve either of the two goals.

\subsubsection{Designing Pure Demand-Sided Intervention Mechanisms for Reducing Government} Interventions

Based on the effectiveness of one- and two-sided intervention mechanisms in Proposition 2, we have the following corollary:

Corollary 1. For ease of implementation, pure demand-sided intervention mechanisms $\left(M^{D}=\left(s^{(\alpha, \beta)}(f), 0\right)\right.$ satisfying $\left.\psi(\alpha, \beta)=-e^{(0,0)}\right)$ should be designed for reducing government interventions relative to two-sided intervention mechanism $M^{D S}=\left(s^{(\alpha, \beta)}(f), e^{(\alpha, \beta)}\right)$ when both can coordinate the vaccine market.

Corollary 1 intuitively indicates that pure demand-sided intervention mechanisms are favored by a government over two-sided intervention mechanisms. This implies that the presence of reference dependence can reduce government interventions in supply side. More importantly, its presence activates pure demand-sided intervention mechanism to maximize the social welfare, which is impossible in Adida, Dey and Mamani [3].

\subsubsection{Designing Specific Two-Sided Intervention Mechanisms for Achieving Budget Neutrality}

In addition to reducing government interventions, reference dependence allows specific two-sided intervention mechanisms to satisfy budget neutrality. To do so, we define a government budget as the total payment (including subsidies and taxes) used for intervention mechanisms, i.e.,

$$
B(\alpha, \beta)=q^{*} \cdot \mathbf{e}^{(\alpha, \beta)}+f^{*} \cdot s^{(\alpha, \beta)}(f)
$$

where the first term reflects the payment (including subsidies or taxes) for the vaccine manufacturer and the second term represents the payment for vaccinated individuals. If the budget of one-sided (or two-sided) mechanisms is denoted as $B^{O M}(\alpha, \beta)$ (or $B^{T M}(\alpha, \beta)$ ), the following proposition can be got:

Proposition 3. Given $M^{D}=\left(s^{(\alpha, \beta)}(f), 0\right)$ and $M^{D S}=\left(s^{(\alpha, \beta)}(f), e^{(\alpha, \beta)}\right)$, we have: the former does not achieve budget neutrality (i.e., $B^{O M}(\alpha, \beta) \neq 0$ ) whereas the latter can, under $B^{T M}(\alpha, \beta)=0$

Intuitively, $M^{D}=\left(s^{(\alpha, \beta)}(f), 0\right)$ fail to achieve an ex-ante balanced budget. For example, a budget deficit occurs when subsidizing vaccinated individuals. Similarly, a budget surplus occurs when taxing vaccinated individuals. However, specific two-sided intervention mechanisms satisfying $B^{T M}(\alpha, \beta)=0$ can be ex-ante budget-neutral. The underlying reason is that, according to the different levels of vaccination coverage and the resulting demand-sided intervention mechanisms (subsidy or tax), a government can choose the 'right' vaccine manufacturer whose reference dependence satisfies $B^{T M}(\alpha, \beta)=0$. In particular, when vaccine coverage is insufficient $(0 \leq f<\bar{F})$ and subsidies are offered to vaccinated individuals, taxes can be imposed on a vaccine manufacturer with reference dependence satisfying $B^{T M}(\alpha, \beta)=q^{*} \cdot e^{(\alpha, \beta)}+f^{*} \cdot \min \left\{s^{(\alpha, \beta)}(f), s^{(\alpha, \beta)}\left(f^{*}\right)\right\}=0$. Alternatively, when vaccine coverage exceeds the critical vaccination fraction $(\bar{F} \leq f \leq 1)$ and taxes are imposed on vaccinated individuals, subsidies are offered to a vaccine manufacturer with reference dependence preference satisfying $B^{T M}(\alpha, \beta)=q^{*} \cdot e^{(\alpha, \beta)}+f^{*} \cdot(-\beta)=0$.

In practice, pure demand-sided intervention mechanisms are extensively used by a government subject to a neutral budget. This is the case because these demand-sided intervention mechanisms are modified to satisfy budget neutrality. That is, a government returns (or collects) additional subsidies (or taxes) $t>0$ (or $t<0$ ) to (or from) all individuals before intervention mechanisms $\left(s^{(\alpha, \beta)}(f), e^{(\alpha, \beta)}\right)[36]$. 
Thus, this modification is added to the sequence of events given in the beginning of Section 3 and is positioned first.

Fortunately, this modification does not induce all players to change their decisions in pure demand-sided intervention mechanisms. Specifically, individuals' choices and vaccine demand do not change because additional subsidies (or taxes) are offered (or imposed) to all individuals, rather than to vaccinated or unvaccinated individuals alone. Due to this fixed vaccine demand, the reference-dependent manufacturer does not adjust their decisions. The following proposition characterizes this modified mechanism.

Proposition 4. Given that $M^{D}=\left(s^{(\alpha, \beta)}(f), 0\right)$ satisfies $\psi(\alpha, \beta)+e^{(0,0)}=0$. To achieve budget neutrality, this mechanism is modified as $M_{t}^{D}=\left(t, s^{(\alpha, \beta)}(f), 0\right)$ where $t$ satisfies $t=-s^{(\alpha, \beta)}(f) f^{*}$.

Proposition 4 implies that under these modified mechanisms, a government can apply two opposing actions. For example, when all individuals are imposed taxes in the first place, vaccinated individuals are subsequently provided to subsidies [41]. These vaccinated individuals are provided to both taxes and subsidies while taxes only are imposed on those unvaccinated. Thus, the instrument of simultaneously imposing taxes and providing subsidies echoes a 'stick-and-carrot' method, which are described in prior papers [36].

From the discussion about two-sided and modified one-sided mechanisms, one can find that both are always ex-ante budget-neutral during the process of maximizing the social welfare. Further, the two mechanisms yield two significant implications. On one hand, budget neutrality fills the gap of a two-part menu given in Adida, Dey and Mamani [3], that are always at a budget deficit to achieve the first best solutions. On the other hand, new management insights are shown in the following proposition.

Proposition 5. Given that modified demand-sided intervention mechanisms $M_{t}^{D}=\left(t, s^{(\alpha, \beta)}(f), 0\right)$ satisfy both $\psi(\alpha, \beta)+e^{(0,0)}=0$ and $t=-s^{(\alpha, \beta)}(f) f^{*}$, and specific two-sided intervention mechanisms $M^{D S}=$ $\left(s^{(\alpha, \beta)}(f), e^{(\alpha, \beta)}\right)$ satisfy both $\psi(\alpha, \beta)+e^{(0,0)} \neq 0$ and $B^{T M}(\alpha, \beta)=0$. Since the two mechanisms maximizing social welfare are ex-ante budget-neutral, both can provide a flexibility to a sophisticated government to arbitrarily allocate surplus between the vaccine manufacturer and unvaccinated individuals.

Proposition 5 indicates that specific two-sided and modified demand-sided mechanisms can be chosen to achieve ex-ante budget neutrality when a government balances $t$ and reference dependence $(\alpha, \beta)$ carefully. This balance depends on the relative emphasis that a government places on the consumer surplus of unvaccinated individuals versus the utility of a reference-dependent manufacturer. If a government adopts modified demand-sided intervention mechanisms, these unvaccinated individuals will be subsidized or taxed while the reference-dependent manufacturer is not directly affected by economic incentives. Alternatively, if specific two-sided intervention mechanisms are adopted, subsidy or tax does not affect the consumer surplus of the unvaccinated individuals but causes the expected utility of the reference-dependent manufacturer to change.

\section{The Negative Impact of Reference Dependence}

Each coin has two sides. Section 4 has shown the positive impact of reference dependence in intervention mechanisms under specific conditions. Next, we will uncover its negative impact on general two-sided intervention mechanisms. For this purpose, we return to Theorem 3 and study that the value of reference dependence satisfies $\psi(\alpha, \beta) \neq-e^{(0,0)}$ and $B^{T M}(\alpha, \beta) \neq 0$.

In general two-sided intervention mechanisms, we can get the two-part menu of subsidies shown in Adida, Dey and Mamani [3] by assuming the special case of $\alpha=0$ and $\beta=0$. By comparing intervention mechanisms in the scenario with and without reference dependence ( $\alpha=\beta=0$ vs. $\alpha>0$ and 
$\beta>0$ ), one can find the contrasting results, implying the negative impact of reference dependence in general two-sided intervention mechanisms.

Proposition 6. We compare general two-sided intervention mechanisms, satisfying Equations (5) and (6), with previous intervention mechanisms $(\alpha=0, \beta=0)$ : The difference in the supply side is $\mathrm{e}^{(\alpha, \beta)}-\mathrm{e}^{(0,0)}=\psi(\alpha, \beta)$ while the counterpart in the demand side depends on the degree of vaccination coverage, i.e.,

$$
s^{(\alpha, \beta)}(f)-s^{(0,0)}(f)= \begin{cases}\alpha N(f), & 0 \leq f<f^{*} \\ \alpha N\left(f^{*}\right), & f^{*} \leq f<\bar{F} \\ -\beta, & \bar{F} \leq f \leq 1\end{cases}
$$

Proposition 6 shows the negative impact of reference dependence, which makes general two-sided intervention mechanism complex. This complexity directly reflects in differences in terms of intervention's extent (Section 5.1) and intervention's structure (Section 5.2).

\subsection{Making General Two-Sided Intervention Mechanisms Complex in Intervention's Extent}

Intervention's extent is characterized by the optimal payment extent in intervention mechanisms (or rather, the size of tax or subsidy). Its difference implies that reference dependence preference yields a change in the extent of the optimal payment, which will be shown in the demand and supply side, respectively.

\subsubsection{Analysis in Supply-Sided Intervention Mechanisms}

In supply-sided intervention mechanisms, Proposition 6 shows that $\psi(\alpha, \beta)$ measures the difference in intervention's extent, or equivalently, the changeable payments caused by reference dependence. In Figure 2 (Values of input parameters can be found in Appendix C), Region (i) and (iii) described by the gap between dotted blue and solid red lines characterize the cases of $\psi(\alpha, \beta)>0$ and $-e^{(0,0)}<\psi(\alpha, \beta)<0$, respectively. Ignoring these differences leads to the over- or under-use of subsidies, which implies a failure to mitigate yield uncertainty that shapes the inefficiency of the vaccine market.

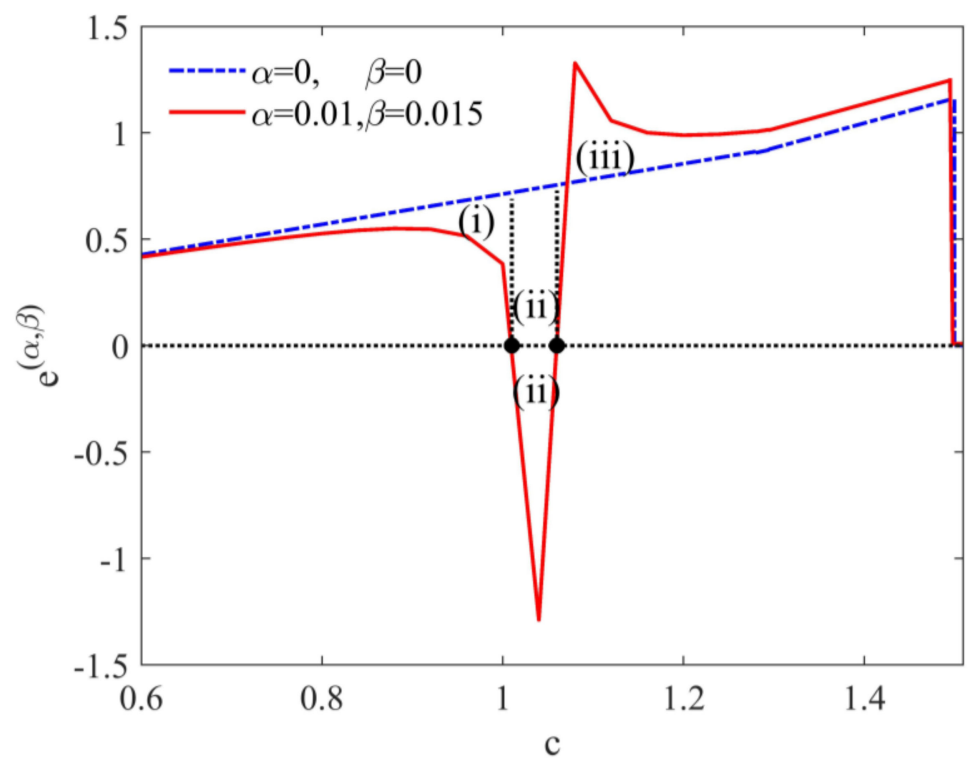

Figure 2. (Color online) supply-sided intervention mechanisms. 


\subsubsection{Analysis in Demand-Sided Intervention Mechanisms}

Before showing the difference in intervention's extent, we first analyze demand-sided intervention mechanism, which depends on vaccination coverage.

First, when $0 \leq f<f^{*}$, vaccination coverage is lower in the decentralized system than in the centralized system as a result of vaccination externality. To fill this gap, heterogeneous subsidies (i.e., $\left.s^{(\alpha, \beta)}(f)=\theta f+\alpha N(f)\right)$ should be provided as a function of the number of vaccinated individuals. Within the range of $0 \leq f<\bar{F}$ in Figure 3 (or $0 \leq f<\bar{U} q^{*}$ in Figure 4), the solid red line characterizes heterogeneous subsidies whereby vaccination coverage achieves the first best solution.

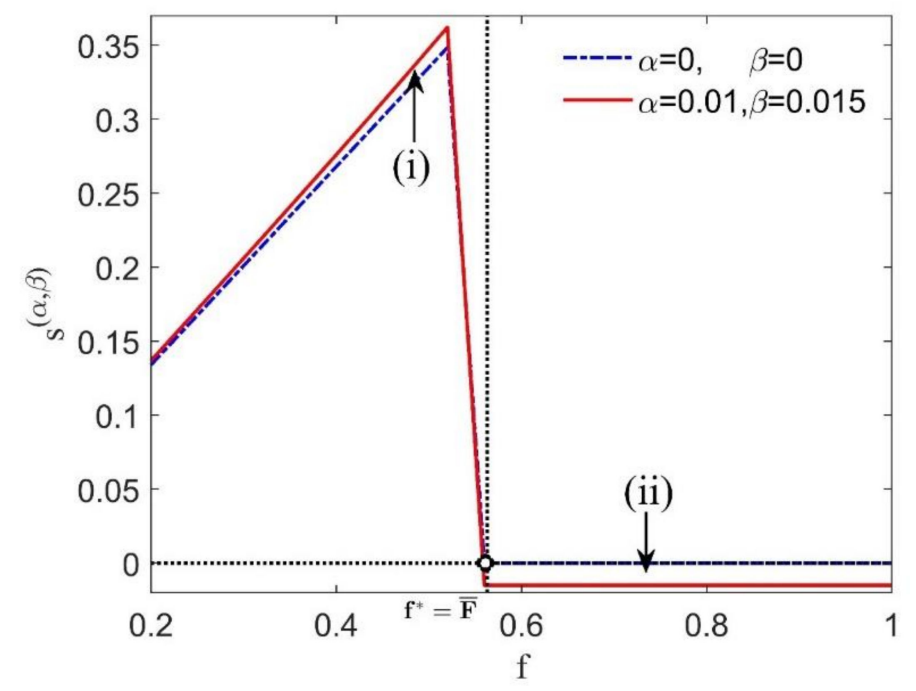

Figure 3. (Color online) demand-sided intervention mechanisms when $f^{*}=\bar{F}$.

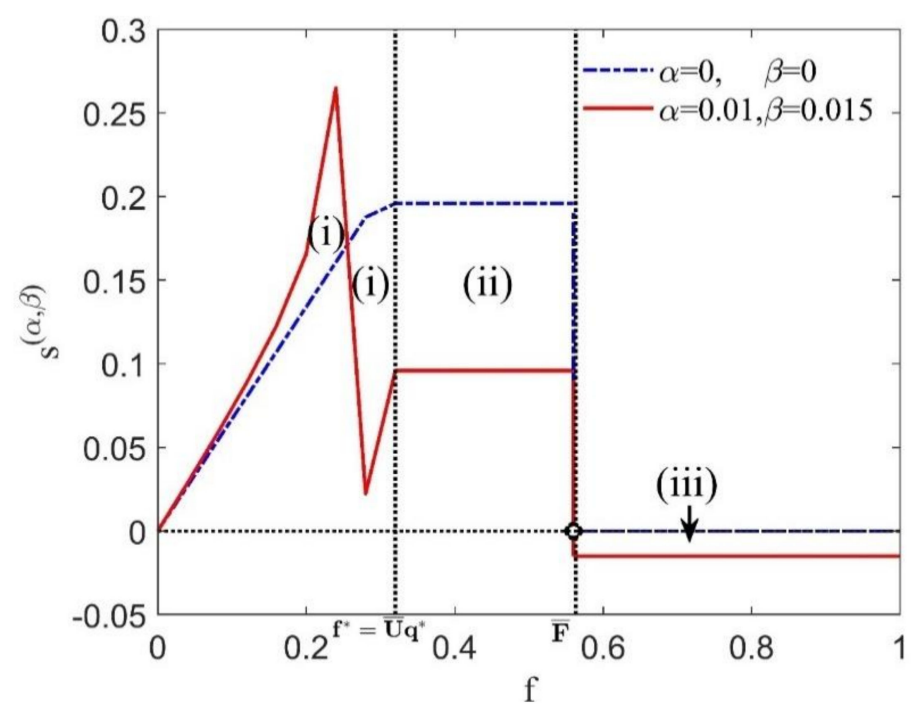

Figure 4. (Color online) demand-sided intervention mechanisms when $f^{*}=\bar{U} q^{*}$.

Second, when $f^{*} \leq f<\bar{F}$, vaccination coverage under the decentralized system ranges between the socially optimal level $\left(f^{*}\right)$ and the required level for herd immunity $(\bar{F})$ (see the solid red line in the range of $\left[\bar{U} q^{*}, \bar{F}\right)$ in Figure 4$)$. In this case, when a government subsidizes an additional individual to get vaccinated, the increment of social welfare is lower than the increment involved with the corresponding social cost (see the proof of Theorem 3). Thus, there is no need to offer excess subsidy to individuals within the range of $\left[f^{*}, \bar{F}\right)$ and the subsidy maintains the fixed magnitude of $\theta f^{*}+\alpha N\left(f^{*}\right)$. 
Third, when $\bar{F} \leq f \leq 1$, vaccination coverage in the decentralized system exceeds the requirement of herd immunity. In this case, to maximize the expected utility, a reference-dependent manufacturer tends to enhance the level of vaccination coverage by inducing individuals with low infection costs to get vaccinated and further to reduce the psychological utility of overproduction (i.e., the term $\beta \bar{J}\left(\frac{f}{q^{*}}\right)$ in the proof of Theorem 3). However, such an induced behavior leads to poor outcomes: the vaccinated fraction exceeding the requirement for herd immunity reduces the social welfare due to an increment in vaccine demand. Due to this, a government should discourage manufacturer's induced behavior by imposing sales taxes to increase the effective vaccine price of vaccinated individuals (i.e., $\left.s^{(\alpha, \beta)}(f)=-\beta\right)$. These taxes are characterized by the solid red lines within the range of $\bar{F} \leq f \leq 1$ shown in Figure 3 (or Figure 4). Besides, Arifoglu and Tang [41] show the different roles of imposed taxes. That is, sales tax discourages individuals with low infection costs from seeking vaccination so that limited vaccines can be allocated to individuals with a high probability of becoming infected. Obviously, such taxes discourage individuals' behavior, rather than the manufacturer's behavior, in our model.

After showing the analysis above, Proposition 6 shows the difference in intervention extent. That is, both yield uncertainty and the psychological cost of underproduction, $\alpha N(f)$ (or $\alpha N\left(f^{*}\right)$ ), measure the changeable payment extent of sales subsidies when $0 \leq f<f^{*}$ (or $f^{*} \leq f<\bar{F}$ ) (the case of $\bar{F} \leq f \leq 1$ will be analyzed later). The two changes, or equivalently, the difference in intervention's extent, can be observed from the gap between solid red and dotted blue lines in Figures 3 and 4 . When a government ignores these changes in intervention's extent, the socially optimal levels of vaccination coverage are not achieved. Taking the range of $0 \leq f<\bar{F}$ given in Figure 4 as an example, ignoring reference dependence in Equation (6) (i.e., the dotted blue line with $\alpha=0$ and $\beta=0$ ), results in that both the psychological cost of underproduction and yield uncertainty have no influences in the intervention mechanisms. Consequently, the optimal subsidy level described by the dotted blue line is under- or over-used relative to the counterpart described by the solid red line. This implies a failure of intervention mechanisms to mitigate vaccination externality that shapes the inefficiency of the vaccine market.

\subsection{Making General Two-Sided Intervention Mechanisms Complex in Intervention's Structure}

Intervention's structure means that the direction of the optimal payment used to coordinate the vaccine market may be positive (subsidy), zero, or negative (tax). Similar with the analysis of differences in intervention's extent, we also adopt a similar way to analyze difference in intervention's structure.

\subsubsection{Analysis in Supply-Sided Intervention Mechanisms}

When comparing the structures between $e^{(\alpha, \beta)}$ and $e^{(0,0)}$ in Theorem 3 (or observing solid red and dotted blue lines in Figure 3), we find their differences: The former are heterogeneous and can be constructed by subsidy $\left(\mathrm{e}^{(\alpha, \beta)}>0\right)$, zero $\left(\mathrm{e}^{(\alpha, \beta)}=0\right)$ or tax $\left(\mathrm{e}^{(\alpha, \beta)}<0\right)$, whereas the latter are homogeneous and include subsidy alone $\left(e^{(0,0)}>0\right)$. This heterogeneity structure depends on the interactions between economic and behavioral issues (i.e., $\psi(\alpha, \beta)$ in Proposition 6). Specifically, both marginal benefits (i.e., $\left.\left[T\left(f^{*}\right)+s\left(f^{*}\right)\right] \int_{0}^{\frac{f^{*}}{q^{*}}} x d J(x)\right)$ and the psychological disutility of underproduction (i.e., $\left.\alpha \int_{0}^{\frac{f^{*}}{q^{*}}} x d J(x)\right)$ shrink the payment of supply-sided mechanisms (i.e., $\mathrm{e}^{(\alpha, \beta)}$ ). Conversely, both production costs (i.e., $c$ ) and the psychological cost of overproduction (i.e., $\beta \int_{\frac{f^{*}}{\bar{q}^{*}}}^{\bar{U}} x d J(x)$ ) increase such payments.

More importantly, subsidy and tax, as a main difference in intervention's structure, have opposite effects. Ignoring reference dependence easily leads to misuse subsidy and tax. For example, Region (ii) in Figure 2 shows that cost subsidy and tax are characterized by dotted blue and solid red lines, respectively. The former (the latter) is designed to enhance (reduce) production quantities of a vaccine manufacturer without (with) reference dependence. When a government ignores reference dependence 
and the resulting difference in intervention's structure, cost subsidy that should be offered to a vaccine manufacturer with $\alpha=0$ and $\beta=0$ is now offered to a vaccine manufacturer with $\alpha=0.01$ and $\beta=0.015$ by mistake. Consequently, the misuse of cost subsidy induces such a reference-dependent manufacturer to enhance vaccine quantities. In fact, this manufacturer should be intervened to reduce vaccine quantity.

\subsubsection{Analysis in Demand-Sided Intervention Mechanism}

When comparing the structures between $s^{(\alpha, \beta)}(f)$ and $s^{(0,0)}(f)$ in Theorem 3, we find their differences: $s^{(\alpha, \beta)}(f)$ is heterogeneous structure, including both subsidy $\left(s^{(\alpha, \beta)}(f)>0\right.$ when $\left.0 \leq f<\bar{F}\right)$ and $\operatorname{tax}\left(s^{(\alpha, \beta)}(f)<0\right.$ when $\left.\bar{F} \leq f \leq 1\right)$, whereas $s^{(0,0)}(f)$ is homogeneous structure, including subsidies alone $\left(s^{(0,0)}(f)>0\right)$. In Figures 3 and 4 , these different structures can be observed in dotted blue and solid red lines. Due to the difference in intervention's structure captured by $-\beta$ in Proposition 6 , the misuse of intervention instruments (subsidy and tax) fails to achieve the first best solution. The reason supporting this result is the same as the analysis in Section 5.2.1.

\section{Conclusions and Future Research}

This paper proposed a unified analysis framework to analyze the implication of reference dependence in vaccine markets. In this framework, we characterized reference dependence preference as the psychological disutility of overproduction and underproduction. Then, this characterization was incorporated into manufacturer's expected utility and further, changed the optimal decisions in terms of production quantities and vaccine prices. More importantly, this preference had significant implications in the design of intervention mechanisms.

The positive impact included two aspects. First, this preference categorized intervention mechanisms into one-sided and two-sided, and then examine their effectiveness. In particular, pure-demand and two-sided intervention mechanisms can maximize social welfare. However, this objective is not achieved through pure supply-sided intervention mechanisms. For example, it is necessary to intervene the Ebola vaccine market by two sides (offering subsidies to both manufacturers and individuals [40]) rather than pure supply side. Second, this preference made some intervention mechanisms (i.e., pure demand-sided and special two-sided) not only maximize social welfare, but also achieve other goals, such as reducing government interventions or achieving budget neutrality. On one hand, pure demand-sided intervention mechanisms could reduce government interventions compared to two-sided intervention mechanisms, if both can maximize the social welfare. This result can be verified by the case of some pharmaceutical firms mentioned in Section 1, such as GSK, Sanofi and Johnson \& Johnson. That is, they offer sufficient vaccine to satisfy individuals' demand voluntarily $[9,42]$. On the other hand, both special two-sided and modified demand-sided intervention mechanisms could achieve budget neutrality [36]. The two mechanisms provided flexibility to a government by arbitrarily allocating social welfare between unvaccinated individuals and a reference-dependent manufacturer.

In addition to the positive impact of reference dependence, its negative impact was that, compared to previous intervention mechanism without reference dependence, this preference caused general two-sided intervention mechanisms to change in intervention's extent and structure. Overlooking these changes led to a reduction in the effectiveness of these intervention mechanisms and thus failed to maximize the social welfare.

Our model has several limitations, which reveal avenues for further study. For instance, our paper assumes that reference dependence is unaffected by subsidy/tax schemes. However, this separability assumption can be extended to a situation in which subsidy/tax schemes and reference dependence may serve as substitutes or complements [43]. All individuals are also assumed to be perfectly rational, and their decisions are made in a single period. These assumptions can be extended to a more realistic setting, where a population is mixed (including rational and nonrational individuals) and their decisions are made over two periods. These extensions may yield more interesting conclusions in mechanism design. 
Author Contributions: Conceptualization: E.C. and F.G.; methodology: E.C. and F.G.; writing-original draft: F.G.; writing-review \& editing: E.C. and F.G.; formal analysis: F.G.; software: F.G.; investigation: E.C.; visualization: F.G.; funding acquisition: E.C.; project administration: E.C.; supervision: E.C. All authors have read and agreed to the published version of the manuscript.

Funding: This research was funded by: (i) The National Natural Science Foundation of China under grants 71420107027 and 71671061; and (ii) The Outstanding Young Foundation of Hunan Province Natural Science under grants 2018JJ1003.

Conflicts of Interest: The authors declare no conflict of interest.

\section{Appendix A}

Proof of Lemma 1. First, for the derivative of

$$
E[U(q, f)]=\int_{0}^{\frac{f}{q}}[T(f) x q-c q] d J(x)+\int_{\frac{f}{q}}^{\bar{u}}[T(f) f-c q] d J(x)+\alpha \int_{0}^{\frac{f}{q}}(x q-f) d J(x)+\beta \int_{\frac{f}{q}}^{\bar{u}}(f-x q) d J(x)
$$

with respect to $q$, we have:

$$
\begin{gathered}
\frac{\partial E[U(q, f)]}{\partial q}=\int_{0}^{\frac{f}{q}}[T(f)+\alpha+\beta] x d J(x)-\beta \mu-c, \text { and } \\
\frac{\partial^{2} E[U(q, f)]}{\partial q^{2}}=-\frac{f^{2}}{q^{3}}[T(f)+\alpha+\beta] j\left(\frac{f}{q}\right)<0 .
\end{gathered}
$$

Let $K(q)=\int_{0}^{\frac{f}{q}}[T(f)+\alpha+\beta] x d J(x)-\beta \mu-c$, and in turn we have:

$$
\begin{gathered}
\lim _{q \rightarrow 0} K(q)=[T(f)+\alpha] \mu-c>0 \text { and } \\
\lim _{q \rightarrow \infty} K(q)=-\beta \mu-c<0 .
\end{gathered}
$$

According to the zero point theorem, a unique production quantity value $\hat{q}_{D}$ maximizes the expected utility and satisfies $K\left(\hat{q}_{D}\right)=0$.

Second, we demonstrate the relationship between $\hat{q}_{D}$ and $\alpha$ (or $\beta$ ):

$$
\begin{gathered}
\frac{\partial K\left(\hat{q}_{D}\right)}{\partial \alpha}=\int_{0}^{\frac{f}{\hat{q}_{D}}} x d J(x)>0, \text { and } \frac{\partial \hat{q}_{D}}{\partial \alpha}=-\frac{\frac{\partial K\left(\hat{q}_{D}\right)}{\partial \alpha}}{\frac{\partial^{2} E\left[\left(u\left(q_{,} f\right)\right]\right.}{\partial q^{2}}}<0 \text { as well as } \\
\frac{\partial K\left(\hat{q}_{D}\right)}{\partial \beta}=\int_{0}^{\frac{f}{\hat{q}_{D}}} x d J(x)-\mu<0, \text { and } \frac{\partial \hat{q}_{D}}{\partial \beta}=-\frac{\frac{\partial K\left(\hat{q}_{D}\right)}{\partial \beta}}{\frac{\partial^{2} E[u(q, f)]}{\partial q^{2}}}<0 .
\end{gathered}
$$

Proof of Theorem 1. First, we assume the perfect vaccine, i.e., $\phi=1$. Then, the vaccine demand equation becomes $w=\bar{F}-f$.

Denote $t=\bar{F}-f$ and $z=\frac{f}{q}$. Then, $f=\bar{F}-t$ and $q=\frac{\bar{F}-f}{z}$. Thus, we can rewrite the expected utility of the vaccine manufacturer as:

$$
E[U(z, t)]=\frac{\bar{F}-t}{z}\left[t \int_{0}^{z} x d J(x)+t z \int_{z}^{\bar{U}} x d J(x)-c+\alpha \int_{0}^{z}(x-z) d J(x)+\beta \int_{z}^{\bar{u}}(z-x) d J(x)\right]
$$


We take the first derivatives with respect to $z$ and $t$ :

$$
\begin{aligned}
\frac{\partial E[U(z, t)]}{\partial z} & =-\frac{\bar{F}-t}{z^{2}}\left[t \int_{0}^{z} x d J(x)-c+\alpha \int_{0}^{z} x d J(x)-\beta \int_{z}^{\bar{U}} x d J(x)\right] \\
\frac{\partial E[U(z, t)]}{\partial z} & =\frac{1}{z}\left\{(\bar{F}-2 t)\left[\int_{0}^{z} x d J(x)+z \int_{z}^{\bar{U}} d J(x)\right]+c\right\} \\
& -\frac{1}{z}\left[\alpha \int_{0}^{z}(x-z) d J(x)+\beta \int_{z}^{\bar{U}}(z-x) d J(x)\right]
\end{aligned}
$$

We define new functions $R(z)$ and $S(t)$ as follows:

$$
\begin{gathered}
R(z)=t \int_{0}^{z} x d J(x)-c+\alpha \int_{0}^{z} x d J(x)-\beta \int_{z}^{\bar{u}} x d J(x) \text { and } \\
S(t)=(\bar{F}-2 t)\left[\int_{0}^{z} x d J(x)+z \int_{z}^{\bar{u}} d J(x)\right]+c-\left[\alpha \int_{0}^{z}(x-z) d J(x)+\beta \int_{z}^{\bar{u}}(z-x) d J(x)\right] .
\end{gathered}
$$

Next, we demonstrate that $R(z)$ has, at most, two roots between zero and its upper limit $(\bar{U})$ when $t$ is applied, such that $S(t)=0$. We note that the roots of $R(z)$ are the same as the roots of $\frac{E[U(z, t)]}{\partial z}$ and vice versa; the same is true for $S(t)$ and $\frac{\partial E[U(z, t)]}{\partial t}$. By defining:

$$
\begin{gathered}
\Theta(z)=\int_{0}^{z} x d J(x)+z \int_{z}^{\bar{u}} d J(x), \text { and } \\
\left.\varphi(z)=\alpha \int_{0}^{z}(x-z) d J(x)+\beta \int_{z}^{\bar{U}}(z-x) d J(x)\right]
\end{gathered}
$$

and setting $S(t)=0$, we have:

$$
t=\frac{1}{2}\left[\bar{F}+\frac{c-\varphi(z)}{\Theta(z)}\right]
$$

By substituting $t$ into the expression for $R(z)$ in Equation (A1), we have:

$$
R(z)=\frac{1}{2}\left[\bar{F}+\frac{c-\varphi(z)}{\Theta(z)}\right] \int_{0}^{z} x d J(x)-c+\alpha \int_{0}^{z} x d J(x)-\beta \int_{z}^{\bar{u}} x d J(x) .
$$

Taking the derivation of $R(z)$, we have:

$$
\begin{aligned}
\frac{d R(z)}{d z}= & \frac{j(z)}{2}\left\{z\left[\frac{c}{\Theta(z)}+\bar{F}\right]-\frac{c}{\Theta^{2}(z) \rho(z)} \int_{0}^{z} x d J(x)\right\} \\
& -\frac{1}{2}\left[\frac{\varphi(z)}{\theta(z)}\right]^{\prime} \int_{0}^{z} x d J(x)+j(z) z\left[\alpha \beta-\frac{\varphi(z)}{2 \theta(z)}\right] \\
& =\frac{j(z)}{2}\left\{Z\left[\frac{c-\varphi(z)}{\theta(z)}+\bar{F}+2(\alpha+\beta)\right]+\frac{M(z)-c-\beta \mu}{\theta_{2}(z) \rho(z)} \int_{0}^{z} x d J(x)\right\}
\end{aligned}
$$

where $\rho(z)=\frac{j(z)}{1-J(z)}$ is the hazard rate of variable $u_{E}$ and $M(z)=\frac{\alpha \int_{0}^{z} x d J(x)}{1-J(z)}$.

The second derivative of $R(z)$ where its first derivation is zero can be written as follows:

$$
\begin{aligned}
\left.\frac{d^{2} R(z)}{d z^{2}}\right|_{\frac{d R(z)}{d z}=} & =\left\{\frac{M^{\prime}(z)}{\Theta^{2}(z) \rho(z)}+\frac{[c+\beta \mu-M(z)] \cdot\left[2 \Theta^{\prime}(z) \rho(z)+\Theta(z) \rho^{\prime}(z)\right]}{\Theta^{3}(z) \rho(z)}\right\} \int_{0}^{z} x d J(x) \\
& +\underbrace{\frac{2 z j(z)[M(z)-c-\beta \mu]}{\theta^{2}(z) p(z)}+\bar{F}+\frac{c-\varphi(z)}{\theta(z)}+2(\alpha+\beta)}_{*} .
\end{aligned}
$$

When Equation (A3) is positive, we have the desired result, whereby $R(z)$ has, at most, two roots. To demonstrate this, we must prove that the portion marked as $(*)$ in Equation (A3) is positive. 
Recall that we set $S(t)=0$ in Equation (A2):

$$
\begin{aligned}
& 0=S(t)=(\bar{F}-2 t) \Theta(z)+c-\varphi(z) \\
& \geq-\bar{F} \Theta(z)+c-\varphi(z) .
\end{aligned}
$$

Thus, $\bar{F} \Theta(\mathrm{z}) \geq c-\varphi(\mathrm{z})$.

We attempt to prove that the portion marked as (*) in Equation (A3) is positive:

$$
\begin{array}{lr}
2 z \bar{j}(z)[M(z)-c-\beta \mu]+\bar{F} \Theta^{2}(z)+[c-\varphi(z)] \Theta(z)+2(\alpha+\beta) \Theta^{2}(z) & \\
\geq 2 z \bar{j}(z)[M(z)-c-\beta \mu]+\bar{F} \Theta(z) z \bar{j}(z)+[c-\varphi(z)] z \bar{j}(z)+2 z \bar{j}(z)(\alpha+\beta) \Theta(z) & (\Theta(z) \geq z \bar{j}(z)) \\
=z \bar{j}(z)\{2[M(z)-c-\beta \mu]+\bar{F} \Theta(z)+[c-\varphi(z)]+2(\alpha+\beta) \Theta(z)\} & \\
\geq z \bar{j}(z)\{2[M(z)-\beta \mu]-2 c+2[c-\varphi(z)]+2(\alpha+\beta) \Theta(z)\} & (\bar{F} \Theta(z) \geq c-\varphi(z)) \\
\geq 2 z \bar{j}(z)\left\{\alpha \int_{0}^{z} x d J(x)-\beta \mu-\alpha \int_{0}^{z} x d J(x)+\alpha z \int_{0}^{z} d J(x)-\beta z \int_{z}^{\bar{u}} d J(x)+\beta \int_{z}^{\bar{u}} x d J(x)\right\} & \\
+2 z \bar{j}(z)\left\{\beta\left[\int_{0}^{z} x d J(x)+z \int_{z}^{\bar{u}} d J(x)\right]+\alpha\left[\int_{0}^{z} x d J(x)+z \int_{z}^{\bar{u}} d J(x)\right]\right\} & \left(M(z) \geq \alpha \int_{0}^{z} x d J(x)\right) \\
=2 z \bar{j}(z)\left\{\alpha z \int_{0}^{z} d J(x)+\alpha\left[\int_{0}^{z} x d J(x)+z \int_{z}^{\bar{u}} d J(x)\right]\right\} & \\
\geq 0 . &
\end{array}
$$

As a result, when distributions satisfy $[c+\beta \mu-M(z)] \cdot\left[2 \Theta^{\prime}(z) \rho(z)+\Theta(z) \rho^{\prime}(z)\right] \geq 0$, then Equation (A3) is nonnegative. Therefore, the derivation of the expected utility has, at most, two roots, with the first being the local maximum and with the second being the local minimum.

With the following steps, we show that the minimum root always falls outside of the feasible range of vaccination fractions. Recalling that $(t+\alpha+\beta) \int_{0}^{z} x d J(x)=c+\beta \mu$ in Lemma 1, we have $\int_{0}^{z} x d J(x)=\frac{c+\beta \mu}{t+\alpha+\beta}$. As $0<\int_{0}^{z} x d J(x) \leq \mu$, we have $0<\frac{c+\beta \mu}{t+\alpha+\beta} \leq \mu$ and then $\frac{c}{\mu}-\alpha<t \leq \bar{F}$.

By substituting $t=\frac{c}{\mu}-\alpha$ into Equation (A1), we have:

$$
\begin{aligned}
R(z) & =\left(\frac{c}{\mu}-\alpha\right) \int_{0}^{z} x d J(x)-c+\alpha \int_{0}^{z} x d J(x)-\beta \int_{z}^{\bar{u}} x d J(x) \\
& =\frac{c}{\mu} \int_{0}^{z} x d J(x)-c-\beta \int_{z}^{\bar{u}} x d J(x)=\frac{c\left(\int_{0}^{z} x d J(x)-\mu\right)}{\mu}-\beta \int_{z}^{\bar{u}} x d J(x)<0 .
\end{aligned}
$$

Second, we define the imperfect vaccine as follows:

$$
T(f)=\theta r(f)= \begin{cases}0, & \text { if } f>\bar{F} \\ \theta\left(1-\phi f-\frac{1}{R_{0}}\right), & \text { otherwise }\end{cases}
$$

Let $t=\theta\left(1-\phi f-\frac{1}{R_{0}}\right)$ and $z=\frac{f}{q}$. Then, $f=\frac{1}{\phi}\left(1-\frac{1}{R_{0}}-\frac{t}{\theta}\right)$ and $q=\frac{f}{z \phi}\left(1-\frac{1}{R_{0}}-\frac{t}{\theta}\right)$. Thus, we can rewrite the objective function as:

$E[U(z, t)]=\frac{1}{z \phi}\left(1-\frac{1}{R_{0}}-\frac{t}{\theta}\right)\left[t \int_{0}^{z} x d J(x)+t z \int_{z}^{\bar{u}} x d J(x)-c+\alpha \int_{0}^{z}(x-z) d J(x)+\beta \int_{z}^{\bar{u}}(z-x) d J(x)\right]$.

The rest of the proof proceeds similarly to the perfect case above.

Proof of Theorem 2. This proof follows the same principles of Theorem 2 described in Adida, Dey and Mamani [3] and is thus omitted for brevity. 
Proof of Proposition 1. First, we take the derivation of the manufacturer's expected utility without intervention mechanism (i.e., $s=0$ and $e=0$ ):

$$
E[U(q, f)]=\int_{0}^{\frac{f}{q}}[T(f) x q-c q] d J(x)+\int_{\frac{f}{q}}^{\bar{u}}[T(f) f-c q] d J(x)+\alpha \int_{0}^{\frac{f}{q}}(x q-f) d J(x)+\beta \int_{\frac{f}{q}}^{\bar{u}}(f-x q)
$$

with respect to $f$ and $q$ :

$$
\begin{aligned}
& \frac{\partial E[U(q, f)]}{\partial q}=T(f) \int_{0}^{\frac{f}{q}} x d J(x)-c+\alpha \int_{0}^{\frac{f}{q}} x d J(x)-\beta \int_{\frac{f}{q}}^{\bar{u}} x d J(x), \text { and } \\
& \frac{\partial E[U(q, f)]}{\partial f}=T^{\prime}(f) q \int_{0}^{\frac{f}{q}} x d J(x)+T^{\prime}(f) f \int_{\frac{f}{q}}^{\bar{u}} d J(x)+T(f) \int_{\frac{f}{q}}^{\bar{u}} d J(x)-\alpha \int_{0}^{\frac{f}{q}} d J(x)+\beta \int_{\frac{f}{q}}^{\bar{u}} d J(x)
\end{aligned}
$$

Second, we assume by contrast that both $f=f^{*}$ and $q=q^{*}$ satisfy optimal values of the decentralized system. Determining the optimality of $q=q^{*}$ for $f=f^{*}$ leads to:

$$
\left.\frac{\partial E\left[U\left(q, f^{*}\right)\right]}{\partial q}\right|_{q=q^{*}}=T\left(f^{*}\right) \int_{0}^{\frac{f^{*}}{q^{*}}} x d J(x)-c+\alpha \int_{0}^{\frac{f^{*}}{q^{*}}} x d J(x)-\beta \int_{\frac{f^{*}}{q^{*}}}^{\bar{u}} x d J(x)=0 .
$$

Then, for optimal quantity volume $q=q^{*}$, we show that the decentralized system does not achieve the optimal level of vaccination coverage without intervention mechanisms. We consider the following two cases: (i) $f^{*}=\bar{F}<\bar{U} q^{*}$ and (ii) $f^{*}=\bar{U} q^{*}<\bar{F}$.

(i) Suppose that $f^{*}=\bar{F}<\bar{U} q^{*}$ and $q=q^{*}$. Recalling the derivative of manufacturer's utility at $f^{*}$ (i.e., Equation (A4)) and $\lim _{f \rightarrow\left(f^{*}\right)^{+}} T(f)=\lim _{f \rightarrow\left(f^{*}\right)^{+}} T^{\prime}(f)=0$, we have:

$$
\left.\frac{\partial E\left[U\left(q^{*}, f\right)\right]}{\partial f}\right|_{f \rightarrow\left(f^{*}\right)^{+}}=-\alpha \int_{0}^{\frac{f}{\bar{q}^{*}}} d J(x)+\beta \int_{\frac{f}{q^{*}}}^{\bar{U}} d J(x) .
$$

If $\beta \int_{\frac{f}{q^{*}}}^{\bar{u}} d J(x)-\alpha \int_{0}^{\frac{f}{q^{*}}} d J(x)=0$, we have $\left.\frac{\partial E\left[U\left(q^{*}, f\right)\right]}{\partial f}\right|_{f \rightarrow\left(f^{*}\right)^{+}}=0$. However, replacing both $f^{*}=\bar{F}$ and $\beta \int_{\frac{f}{q^{*}}}^{\bar{u}} d J(x)-\alpha \int_{0}^{\frac{f}{q^{*}}} d J(x)=0$ into Equation (A5), we get $\left.\frac{\partial E[U(q, \bar{F})]}{\partial q}\right|_{q=q^{*}}=T(\bar{F}) \int_{0}^{\bar{u}} x d J(x)-c=$ $-c<0$. This inequation runs contrary to the optimality conditions of Equation (A5). As a result, the optimal level of vaccination coverage achieved under a decentralized system is not the same as $f^{*}=\bar{F}$.

(ii) Supposing that $f^{*}=\bar{U} q^{*}<\bar{F}$ and $q=q^{*}$, we have:

$$
\left.\frac{\partial E\left[U\left(q^{*}, f\right)\right]}{\partial f}\right|_{f \rightarrow\left(f^{*}\right)^{-}}=T^{\prime}\left(\bar{U} q^{*}\right) q^{*} \int_{0}^{\bar{U}} x d J(x)-\alpha<0 .
$$

The left derivative of the manufacturer's expected utility at $f^{*}$ is negative due to $T^{\prime}\left(\bar{U} q^{*}\right)<0$, and thus, the optimal level of vaccination coverage achieved in the decentralized system is not the same as $f^{*}=\bar{U} q^{*}$.

Therefore, these optimal values, $f=f^{*}$ and $q=q^{*}$, under the centralized system are not the same as those achieved under the decentralized system. 
Proof of Theorem 3. Under both $\mathrm{e}^{(\alpha, \beta)}$ and $s^{(\alpha, \beta)}(f)$, we have the manufacturer's expected utility:

$$
\begin{gathered}
E\left[U^{D S}(q, f)\right]=\int_{0}^{\frac{f}{q}}\left[T(f)+s^{(\alpha, \beta)}(f)\right] x q d J(x)+\int_{\frac{f}{q}}^{\bar{u}}\left[T(f)+s^{(\alpha, \beta)}(f)\right] f d J(x) \\
-\left(c-\mathrm{e}^{(\alpha, \beta)}\right) q+\alpha \int_{0}^{\frac{f}{q}}(x q-f) d J(x)+\beta \int_{\frac{f}{q}}^{\bar{u}}(f-x q) d J(x) .
\end{gathered}
$$

Take the derivation of the manufacturer's expected utility with respect to $f$ and $q$ :

$$
\begin{aligned}
& \frac{\partial E\left[U^{D S}(q, f)\right]}{\partial q}= {\left[T(f)+s^{(\alpha, \beta)}(f)+\alpha+\beta\right] \int_{0}^{\frac{f}{q}} x d J(x)-c+e^{(\alpha, \beta)}-\beta \mu, \text { and } } \\
& \frac{\partial E\left[U^{D S}(q, f)\right]}{\partial f}=\left[\frac{\partial T(f)}{\partial f}+\frac{\partial s^{(\alpha, \beta)}(f)}{\partial f}\right] q \int_{0}^{\frac{f}{q}} x d J(x)+\left[\frac{\partial T(f)}{\partial f}+\frac{\partial s^{(\alpha, \beta)}(f)}{\partial f}\right] f \int_{\frac{f}{q}}^{\bar{u}} d J(x) \\
&+\left[T(f)+s^{(\alpha, \beta)}(f)\right] \int_{\frac{f}{q}}^{\bar{u}} d J(x)+\beta \int_{\frac{f}{q}}^{\bar{u}} d J(x)-\alpha \int_{0}^{\frac{f}{q}} d J(x)
\end{aligned}
$$

We demonstrate that socially optimal values including $f=f^{*}$ and $q=q^{*}$ will satisfy optimality conditions of the decentralized system. Demonstrating the optimality of $q=q^{*}$ for $f=f^{*}$ is simply written as:

$$
\left.\frac{\partial E\left[U^{D S}\left(q, f^{*}\right)\right]}{\partial q}\right|_{q=q^{*}}=\left[T\left(f^{*}\right)+s^{(\alpha, \beta)}\left(f^{*}\right)+\alpha+\beta\right] \int_{0}^{\frac{f^{*}}{q}} x d J(x)-c+\mathrm{e}^{(\alpha, \beta)}-\beta \mu=0 .
$$

Next, we show that $f^{*}$ is the optimal level of vaccine demand for $q=q^{*}$. For this purpose, we demonstrate that $\left.\frac{\partial E\left[u^{D S}\left(q^{*}, f\right)\right]}{\partial f}\right|_{f<f^{*}}>0$ and $\left.\frac{\partial E\left[u^{D S}\left(q^{*}, f\right)\right]}{\partial f}\right|_{f>f^{*}} \leq 0$.

On one hand, recalling Equation (A6), we have:

$$
\begin{aligned}
\left.\frac{\partial E\left[U^{D S}\left(q^{*}, f\right)\right]}{\partial f}\right|_{f<f^{*}} & =\left[\frac{\partial T(f)}{\partial f}+\frac{\partial_{s}^{(\alpha, \beta)}(f)}{\partial f}\right] f^{*} \int_{0}^{\frac{f}{q^{*}}} x d J(x)+\left[\frac{\partial T(f)}{\partial f}+\frac{\partial_{s}^{(\alpha, \beta)}(f)}{\partial f}\right] \int_{\frac{f}{q^{*}}}^{\bar{U}} f d J(x) \\
& +\left[T(f)+s^{(\alpha, \beta)}(f)\right] \int_{\frac{f}{q^{*}}}^{\bar{U}} d J(x)+\beta \int_{\frac{f}{q^{*}}}^{\bar{u}} d J(x)-\alpha \int_{0}^{\frac{f}{q^{*}}} d J(x) \\
& =q^{*}\left[\theta(1-\phi)+\alpha \frac{\partial N(f)}{\partial f}\right] \int_{0}^{\frac{f}{q^{*}}} x d J(x)+\left[\theta f+\alpha N(f)+\theta\left(1-\phi f-\frac{1}{R_{0}}\right)\right] \int_{\frac{f}{q^{*}}}^{\bar{u}} d J(x) \\
& +f\left[\theta(1-\phi)+\alpha \frac{\partial N(f)}{\partial f}\right] \int_{\frac{f}{q^{*}}}^{\bar{U}} f d J(x)+\beta \int_{\frac{f}{q^{*}}}^{\bar{U}} d J(x)-\alpha \int_{0}^{\frac{f}{q^{*}}} d J(x) \\
& \geq \alpha N(f) \int_{\frac{f}{q^{*}}}^{\bar{U}} d J(x)-\alpha \int_{0}^{\frac{f}{q^{*}}} d J(x) \\
& =\alpha \frac{\mathrm{J}\left(\frac{f}{q^{*}}\right)}{\overline{\mathrm{J}}\left(\frac{f}{q^{*}}\right)-\Delta} \overline{\mathrm{J}}\left(\frac{f}{q^{*}}\right)-\alpha \mathrm{J}\left(\frac{f}{q^{*}}\right) \\
& >\alpha \frac{\mathrm{J}\left(\frac{f}{q^{*}}\right)}{\overline{\mathrm{J}}\left(\frac{f}{q^{*}}\right)} \overline{\mathrm{J}}\left(\frac{f}{q^{*}}\right)-\alpha \mathrm{J}\left(\frac{f}{q^{*}}\right)=0
\end{aligned}
$$

On the other hand, we have:

$$
\left.\frac{\partial E\left[U^{D S}\left(q^{*}, f\right)\right]}{\partial f}\right|_{f>f^{*}}= \begin{cases}-\alpha \int_{0}^{\frac{f}{q^{*}}} d J(x)<0, & \text { if } \bar{F}<\bar{U} q^{*} \\ T^{\prime}\left(\bar{U} q^{*}\right) q^{*} \mu-\alpha<0, & \text { if } \bar{F}>\bar{U} q^{*} .\end{cases}
$$


We note that in the case of $f^{*}=\bar{F}<\bar{U} q^{*}$, we have $T(\bar{F})=T^{\prime}(\bar{F})=s^{\prime}(\bar{F})=0, s(\bar{F})=-\beta$ for all $f>\bar{F}$. For $f^{*}=\bar{U} q^{*}<\bar{F}$, we have $\int_{\bar{q}^{*}}^{\bar{U}} f d J(x)=\int_{\bar{U}}^{\bar{U}} f d J(x)=0$ and $T^{\prime}\left(\bar{U} q^{*}\right)+s^{\prime}\left(\bar{U} q^{*}\right)=T^{\prime}\left(\bar{U} q^{*}\right)<0$ for $f>\bar{U} q^{*}$. Therefore, $f=f^{*}$ for $q=q^{*}$ leads to the desired results.

Proof of Proposition 2. First, we demonstrate the effectiveness of $M^{S}=\left(0, \mathrm{e}^{(\alpha, \beta)}\right)$. Under these conditions, the expected utility of a reference-dependent manufacturer is:

$$
\begin{aligned}
E\left[U^{S}(q, f)\right]= & \int_{0}^{\frac{f}{q}} T(f) x q d J(x)+\int_{\frac{f}{q}}^{\bar{u}} T(f) f d J(x)-\left(c-\mathrm{e}^{(\alpha, \beta)}\right) q \\
& +\alpha \int_{0}^{\frac{f}{q}}(x q-f) d J(x)+\beta \int_{\frac{f}{q}}^{\bar{u}}(f-x q) d J(x)
\end{aligned}
$$

Taking the derivation of the expected utility with respect to $f$ and $q$ :

$$
\begin{aligned}
& \frac{\partial E\left[u^{S}(q, f)\right]}{\partial q}=T(f) \int_{0}^{\frac{f}{q}} x d J(x)-c+\mathrm{e}^{(\alpha, \beta)}+\alpha \int_{0}^{\frac{f}{q}} x d J(x)-\beta \int_{\frac{f}{q}}^{\bar{u}} x d J(x), \text { and } \\
& \frac{\partial E\left[u^{S}(q, f)\right]}{\partial f}=T^{\prime}(f) q \int_{0}^{\frac{f}{q}} x d J(x)+T^{\prime}(f) f \int_{\frac{f}{q}}^{\bar{u}} d J(x)+T(f) \int_{\frac{f}{q}}^{\bar{u}} d J(x)-\alpha \int_{0}^{\frac{f}{q}} d J(x)+\beta \int_{\frac{f}{q}}^{\bar{u}} d J(x) .
\end{aligned}
$$

We assume by contrast that $f=f^{*}$ and $q=q^{*}$ satisfy optimality conditions for the decentralized case. Determining the optimality of $q=q^{*}$ for $f=f^{*}$ leads to:

$$
\left.\frac{\partial E\left[U^{S}\left(q, f^{*}\right)\right]}{\partial q}\right|_{q=q^{*}}=T\left(f^{*}\right) \int_{0}^{\frac{f^{*}}{\bar{q}^{*}}} x d J(x)-c+\mathrm{e}^{(\alpha, \beta)}+\alpha \int_{0}^{\frac{f^{*}}{q^{*}}} x d J(x)-\beta \int_{\frac{f^{*}}{q^{*}}}^{\bar{u}} x d J(x)=0
$$

Then, we show that the reference-dependent manufacturer does not apply the optimal vaccination coverage by considering the following two cases: (i) $f^{*}=\bar{F}<\bar{U} q^{*}$ and (ii) $f^{*}=\bar{U} q^{*}<\bar{F}$. The rest of the proof proceeds similarly to that of Proposition 1 . Therefore, $M^{S}=\left(0, e^{(\alpha, \beta)}\right)$ fails to achieve the socially optimal values.

Second, we demonstrate the effectiveness of $M^{D}=\left(s^{(\alpha, \beta)}(f), 0\right)$ when $\psi(\alpha, \beta)=-e^{(0,0)}$. Under these conditions, the expected utility of a reference-dependent manufacturer is:

$$
\begin{aligned}
E\left[U^{D}(q, f)\right] & =\int_{0}^{\frac{f}{q}}\left[T(f)+s^{(\alpha, \beta)}(f)\right] x q d J(x)+\int_{\frac{f}{q}}^{\bar{u}}\left[T(f)+s^{(\alpha, \beta)}(f)\right] f d J(x)-c q \\
& +\alpha \int_{0}^{\frac{f}{q}}(x q-f) d J(x)+\beta \int_{\frac{f}{q}}^{\bar{u}}(f-x q) d J(x)
\end{aligned}
$$

Taking the derivation of the manufacturer's expected utility with respect to $f$ and $q$ :

$$
\begin{aligned}
\frac{\partial E\left[U^{D}(q, f)\right]}{\partial q} & =\left[T(f)+s^{(\alpha, \beta)}(f)+\alpha+\beta\right] \int_{0}^{\frac{f}{q}} x d J(x)-c-\beta \mu, \text { and } \\
\frac{\partial E\left[U^{D}(q, f)\right]}{\partial f} & =\left[\frac{\partial T(f)}{\partial f}+\frac{\partial s^{(\alpha, \beta)}(f)}{\partial f}\right] q \int_{0}^{\frac{f}{q}} x d J(x) \\
& +\left[\frac{\partial T(f)}{\partial f}+\frac{\partial s^{(\alpha, \beta)}(f)}{\partial f}\right] f \int_{\frac{f}{\bar{q}}}^{\bar{u}} d J(x)+\left[T(f)+s^{(\alpha, \beta)}(f)\right] \int_{\frac{f}{q}}^{\bar{u}} d J(x)+\beta \int_{\frac{f}{\bar{q}}}^{\bar{u}} d J(x)-\alpha \int_{0}^{\frac{f}{\bar{q}}} d J(x) .
\end{aligned}
$$

As $\psi(\alpha, \beta)=-e^{(0,0)}$, we have $\frac{\partial E\left[u^{D}(q, f)\right]}{\partial q}=0$. We then show that the reference-dependent manufacturer can choose the optimal level of vaccination coverage by considering the following two cases: (i) $f^{*}=\bar{F}<\bar{U} q^{*}$ and (ii) $f^{*}=\bar{U} q^{*}<\bar{F}$. The rest of the proof proceeds similarly to that of Proposition 1 . Therefore, $M^{D}=\left(s^{(\alpha, \beta)}(f), 0\right)$ can achieve the socially optimal values. 
Third, we demonstrate the effectiveness of $M^{D S}=\left(s^{(\alpha, \beta)}(f), \mathrm{e}^{(\alpha, \beta)}\right)$ when $\psi(\alpha, \beta) \neq-e^{(0,0)}$. Since the process of this proof is similar to that of Theorem 3, we do not describe the process herein the interest of brevity.

Proof of Corollary 1. This proof process is obvious and we omit it.

Proof of Proposition 3. According to $M^{D}=\left(s^{(\alpha, \beta)}(f), 0\right)$ and $M^{D S}=\left(s^{(\alpha, \beta)}(f), \mathrm{e}^{(\alpha, \beta)}\right)$ given in Theorem 3, we have $B^{O M}(\alpha, \beta)=f^{*} \cdot s^{(\alpha, \beta)}(f)$ and $B^{T M}(\alpha, \beta)=q^{*} \mathrm{e}^{(\alpha, \beta)}+f^{*} s^{(\alpha, \beta)}(f)$. As $s^{(\alpha, \beta)}(f) \neq 0$, we have $B^{O M}(\alpha, \beta) \neq 0$. When $q^{*} \mathrm{e}^{(\alpha, \beta)}+f^{*} \mathcal{S}^{(\alpha, \beta)}(f)=0$, we have $B^{T M}(\alpha, \beta)=0$.

Proof of Proposition 4. Under one-sided mechanisms $M^{D}=\left(s^{(\alpha, \beta)}, 0\right)$, the government budget is $B^{O M}(\alpha, \beta)=s^{(\alpha, \beta)}(f) f^{*}$ where $s^{(\alpha, \beta)}(f)$ given in Theorem 3 . To achieve budget neutrality $B^{O M}(\alpha, \beta)=$ $t+s^{(\alpha, \beta)}(f) f^{*}=0$, additional subsidies (or taxes) $t>0$ (or $t<0$ ) are returned (or collected) to (or from) all individuals. Under this context, $t=-s^{(\alpha, \beta)}(f) f^{*}$.

Proof of Proposition 5. Based on Propositions 3 and 4, we know that specific two-sided and modified one-sided mechanisms are ex-ante budget-neutral and maximize social welfare. The two mechanisms are different in allocating social welfare between the vaccine manufacturer and unvaccinated individuals, affording the flexibility of a government to choose.

Proof of Proposition 6. When $\alpha=0$ and $\beta=0$, demand- and supply-sided intervention mechanisms can be rewritten as:

$$
\begin{gathered}
\mathrm{e}^{(0,0)}=c-\left[T\left(f^{*}\right)+s\left(f^{*}\right)\right] \int_{0}^{\frac{f^{*}}{q^{*}}} x d J(x) \text { and } \\
s^{(0,0)}(f)= \begin{cases}\theta f, & \text { if } 0 \leq f<f^{*}, \\
\theta f^{*}, & \text { if } f^{*} \leq f<\bar{F}, \\
0, & \text { if } \bar{F} \leq f \leq 1,\end{cases}
\end{gathered}
$$

Then, by comparing $s^{(\alpha, \beta)}(f)$ with $s^{(0,0)}(f)$, we have the result in Proposition 6. Similarly, by comparing $\mathrm{e}^{(\alpha, \beta)}$ with $\mathrm{e}^{(0,0)}$, we have the corresponding result in Proposition 6 .

\section{Appendix B}

Before showing this extension, we should technically make the following assumptions:

(1) $\max \{\varphi+\tau-\beta, \tau\} \leq \alpha$;

(2) $\quad 0 \leq(\varphi-\beta) \int_{\frac{f}{q}}^{\bar{u}} d J(x)+(\alpha-\tau) \int_{0}^{\frac{f}{q}} d J(x)<\frac{1}{2}(\phi-\theta \phi \bar{F}) \int_{\frac{f}{q}}^{\bar{u}} d J(x)$;

(3) $c-\mu\left[\phi \lambda+\frac{\phi+T(0)}{2}+\alpha-\tau-\beta+\varphi\right]>0$.

Recalling the total social welfare in Section 3.3.1, we have:

$$
\begin{aligned}
\text { Total social welfare }= & \sigma_{M}+\sigma_{V}+\sigma_{N}+\sigma_{s}=\frac{r(0)}{2}-\frac{(1-g) r(g)+g p(g)}{2}-\lambda r(g)-c q \\
& +(\tau-\alpha) \cdot \max \left(f-\mathbf{u}_{E} q, 0\right)+(\varphi-\beta) \cdot \max \left(\mathbf{u}_{E} q-f, 0\right)
\end{aligned}
$$

where $g=\min \left\{\mathbf{u}_{E} q, f\right\}$ captures the vaccinated fraction of the population. Maximizing this total social welfare is equivalent to minimizing the following social cost:

$$
\begin{aligned}
S C(q, f, U) & =\frac{1}{2}[(2 \lambda+1) r(g)-g T(g)]+c q+(\alpha-\tau) \cdot \max \left(f-\mathbf{u}_{E} q, 0\right) \\
& +(\beta-\varphi) \cdot \max \left(\mathbf{u}_{\mathrm{E}} q-f, 0\right)
\end{aligned}
$$


Thus, first best solution is to minimize this expected social cost function:

$$
\begin{aligned}
\Gamma(q, f) & =\mathrm{E}_{u_{E}}[S C(q, f)] \\
& =\mathrm{E}_{u_{E}}\left[\frac{1}{2}\left((2 \lambda+1) r\left(\min \left\{u_{E} q, f\right\}\right)-\min \left\{u_{E} q, f\right\} T\left(\min \left\{u_{E} q, f\right\}\right)\right)\right. \\
& \left.+c q+(\alpha-\tau) \cdot \max \left(f-\mathbf{u}_{E} q, 0\right)+(\beta-\varphi) \cdot \max \left(\mathbf{u}_{E} q-f, 0\right)\right] \\
& =\int_{0}^{\frac{f}{q}} \frac{1}{2}[(2 \lambda+1) r(x q)-x q T(x q)] d J(x)+\int_{\frac{f}{q}}^{\bar{u}} \frac{1}{2}[(2 \lambda+1) r(f)-f T(f)] d J(x)+c q \\
& +(\alpha-\tau) \int_{0}^{\frac{f}{q}}(f-x q) d J(x)+(\beta-\varphi) \int_{\frac{f}{q}}^{\bar{u}}(x q-f) d J(x)
\end{aligned}
$$

Let $q$ be the production quantity. We first prove that the derivative of $\Gamma(q, f)$ with respect to $f$ is always non-positive when $0 \leq f \leq \bar{F}$. Recall the overall fraction of infected individuals in the population:

$$
r(f)= \begin{cases}0, & \text { if } f>F=\frac{R_{0}-1}{\phi R_{0}} \\ 1-\phi f-\frac{1}{R_{0}}, & \text { otherwise }\end{cases}
$$

If $0 \leq f \leq \bar{F}$, we have:

$$
\frac{\partial \Gamma}{\partial f}=\frac{1}{2}\left[(2 \lambda+1) r^{\prime}(f)-T(f)-f T^{\prime}(f)\right] \int_{\frac{f}{q}}^{\bar{u}} d J(x)+(\varphi-\beta) \int_{\frac{f}{q}}^{\bar{u}} d J(x)+(\alpha-\tau) \int_{0}^{\frac{f}{q}} d J(x)
$$

In this equation, we first consider the term:

$$
\begin{aligned}
& (2 \lambda+1) r^{\prime}(f)-T(f)-f T^{\prime}(f) \\
& \leq-\left(\phi+T(\bar{F})+\bar{F} T^{\prime}(\bar{F})\right) \\
& =-\left(\phi+\bar{F} T^{\prime}(\bar{F})\right) \\
& \leq-(\phi-\theta \phi \bar{F}) .
\end{aligned}
$$

Then, recall Assumption (2), we have:

$$
\begin{gathered}
\frac{\partial \Gamma}{\partial f}=\frac{1}{2}\left[(2 \lambda+1) r^{\prime}(f)-T(f)-f T^{\prime}(f)\right] \int_{\frac{f}{\bar{q}}}^{\bar{u}} d J(x)+(\varphi-\beta) \int_{\frac{f}{\bar{q}}}^{\bar{U}} d J(x)+(\alpha-\tau) \int_{0}^{\frac{f}{q}} d J(x) \\
\leq-\frac{1}{2}(\phi-\theta \phi \bar{F}) \int_{\frac{f}{q}}^{\bar{u}} d J(x)+(\varphi-\beta) \int_{\frac{f}{q}}^{\bar{u}} d J(x)+(\alpha-\tau) \int_{0}^{\frac{f}{q}} d J(x)<0 .
\end{gathered}
$$

Next, we consider two cases: $\bar{F} \leq q \bar{U}$ and. For the first case, $T(f)=0$ if $\bar{F}<f \leq q \bar{U}$. Recall assumption (2), we find $\frac{\partial \Gamma}{\partial f}=(\varphi-\beta) \int_{\frac{f}{q}}^{\bar{u}} d J(x)+(\alpha-\tau) \int_{0}^{\frac{f}{q}} d J(x) \geq 0$ and this implies $f^{*}=\bar{F}$. For the second case, however, we have $\frac{\partial \Gamma}{\partial f}=(\alpha-\tau) \int_{0}^{\frac{f}{q}} d J(x)$ if . Recall assumption (1), we get $\frac{\partial \Gamma}{\partial f} \geq 0$ and this implies $f^{*}=\bar{U} q$.

From the two cases above, the optimal vaccination fraction is $f^{*}=\min \{\bar{F}, \bar{U} q\}$.

To find the optimal $q$, we can simply replace $f$ by $\bar{F}$ in the expression of $\Gamma(q, f)$ and achieve the same objective although $f^{*}=\min \{\bar{F}, \bar{U} q\}$. Then, the objective function is written as a function of $q$. Taking the first and second derivatives of the objective with respect to $q$ leads to: 


$$
\begin{aligned}
& \frac{\partial \Gamma}{\partial q}=-\int_{0}^{\frac{\bar{F}}{q}} \frac{1}{2}\left[\phi(2 \lambda+1)+T(x q)+x q T^{\prime}(x q)\right] x d J(x)+c+(\tau-\alpha) \int_{0}^{\frac{f}{q}} x d J(x)+(\beta-\varphi) \int_{\frac{f}{q}}^{\bar{U}} x d J(x), \text { and } \\
& \frac{\partial^{2} \Gamma}{\partial q^{2}}=-\int_{0}^{\frac{\bar{F}}{q}}\left[\frac{d}{d x}\left(T(x q)+x q T^{\prime}(x q)\right)\right] \frac{x}{2} d J(x)+\frac{1}{2}\left[\phi(2 \lambda+1)+T(\bar{F})+\bar{F} T^{\prime}(\bar{F})+\beta+\alpha-\varphi-\tau\right]\left(\frac{\bar{F}^{2}}{q^{3}}\right) j\left(\frac{\bar{F}}{q}\right) .
\end{aligned}
$$

Since the function $f T(f)$ is concave, one gets $\frac{d}{d x}\left[T(x q)+x q T^{\prime}(x q)\right] \leq 0$. Furthermore, recall assumption (1), we have $\phi+T(\bar{F})+\bar{F} T^{\prime}(\bar{F})+\beta+\alpha-\varphi-\tau \geq 0$ and this inequation makes $\frac{\partial^{2} \Gamma}{\partial q^{2}} \geq 0$. Thus, if $-\mu\left[\phi \lambda+\frac{\phi+T(0)}{2}+\alpha-\tau-\beta+\varphi\right]>0$, then the term $\Gamma(q, f)$ increases over $q \geq 0$, and hence $q^{*}=0$. Otherwise, $q^{*}$ can be got by solving the first order condition:

$$
\int_{0}^{\frac{\bar{F}}{q}} \frac{1}{2}\left(\phi(2 \lambda+1)+T(x q)+x q T^{\prime}(x q)\right) x d J(x)=c+(\tau-\alpha) \int_{0}^{\frac{f}{q}} x d J(x)+(\beta-\varphi) \int_{\frac{f}{q}}^{\bar{u}} x d J(x) .
$$

\begin{tabular}{|c|c|}
\hline \multicolumn{2}{|r|}{ Vaccine Market } \\
\hline$L$ & Constant that determines the absolute value \\
\hline$w$ & Vaccine price \\
\hline$u_{E}$ & Stochastically proportional yield \\
\hline $\bar{U}$ & Upper limit of random yields \\
\hline$\mu$ & Mean value of the random variable of yields \\
\hline$q$ & Target production level \\
\hline$q_{v}$ & Final production output of vaccines \\
\hline$c$ & Unit of the normalized cost of production \\
\hline$\alpha($ or $\beta)$ & Psychological per-unit cost of underproduction (or overproduction) \\
\hline $\begin{array}{l}U(q, w) \text { or } \\
U(q, f)\end{array}$ & Manufacturer's utility \\
\hline $\begin{array}{l}E[U(q, w)] \text { or } \\
E[U(q, f)]\end{array}$ & Expected utility of a vaccine manufacturer with reference dependence \\
\hline $\mathrm{E}[\pi(q, w)]$ & Expected utility of a profit-driven manufacturer \\
\hline$E\left[U_{R}(q, w)\right]$ & Expected psychological disutility of reference dependence \\
\hline$q_{D}^{*}\left(\right.$ or $\left.f_{D}^{*}\right)$ & Optimal production (or vaccination coverage) in the decentralized system \\
\hline$w_{D}$ & Vaccine price in the decentralized system \\
\hline$\rho(z)$ & Hazard rate for yield uncertainty, $\rho(z)=\frac{j(z)}{\int_{z}^{\bar{u}} d J(x)}$ \\
\hline$g$ & $g=\min \left\{\mathrm{u}_{E} q, f\right\}$ \\
\hline$\sigma_{M}$ & Manufacturer's surplus \\
\hline$\sigma_{V}\left(\right.$ or $\left.\sigma_{N}\right)$ & Vaccinated (or unvaccinated) individuals' surplus \\
\hline$\sigma_{S}$ & Other surplus \\
\hline$\tau(\operatorname{or} \varphi)$ & Per-unit benefit from underproduction (or overproduction) \\
\hline$q^{*}\left(\right.$ or $\left.f^{*}\right)$ & Socially optimal production quantity (or vaccination coverage) \\
\hline$\lambda$ & Indirect cost to society \\
\hline$s^{(\alpha, \beta)}(f)$ & A purchase subsidy offered to individuals \\
\hline$e^{(\alpha, \beta)}$ & A cost subsidy per unit provided to the vaccine manufacturer \\
\hline$E^{M}[U(q, f)]$ & Expected utility of the manufacturer with mechanism $M=(s, e)$. \\
\hline$N(f)$ & $N(f)=\frac{J\left(\frac{f}{q^{*}}\right)}{\bar{J}\left(\frac{f}{q^{*}}\right)-\Delta}$ and $\Delta$ satisfies $s^{(\alpha, \beta)}(f)>0$ \\
\hline
\end{tabular}

\section{Appendix C}

Table A1. Notational symbols and their meanings. 
Table A1. Cont.

\begin{tabular}{lc}
\hline & Vaccine Market \\
\hline$\psi(\alpha, \beta)$ & $\psi(\alpha, \beta)=c+\beta \mu-\left[T\left(f^{*}\right)+s\left(f^{*}\right)+\alpha+\beta\right] \int_{0}^{f^{*}} x d J(x)$ \\
$M^{S}\left(\right.$ or $\left.M^{D}\right)$ & One-sided intervention mechanisms on the demand (or supply) side \\
$M^{D S}$ & Two-sided intervention mechanisms \\
$M_{t}^{D}$ & Modified one-sided intervention mechanisms \\
$t$ & Subsidies (or taxes) are offered (or imposed) for all individuals in modified one-sided \\
& intervention mechanisms \\
\hline$R_{0}$ & Infection Transmission \\
$r(f)$ & Basic reproduction number \\
$f$ & Infection probability for the entire population \\
$\phi$ & Fraction of the population to vaccinate and $f=1-u$ \\
$F$ & Degree of vaccine efficacy \\
$p(f)$ or & Critical vaccination fraction, $\bar{F}=$ min $\{F, 1\}$ \\
$P(f)$ & Infection probability level for vaccinated or unvaccinated fractions \\
$\eta$ & A constant that can be adjusted to satisfy $p(f)=\eta(1-\phi) r(f)$ \\
$u$ & A relative loss in infection disutility for the individual \\
$\theta$ & $\bar{u}=1-f$ \\
$T(f)$ & Let $\theta=1-\eta(1-\phi)$ \\
\hline & $T(f) \equiv r(f)-p(f)$ \\
\hline
\end{tabular}

Table A2. Values of input parameters.

\begin{tabular}{lcc}
\hline Parameter & Value & Source \\
\hline$\lambda$ & 1 & Adida, Dey and Mamani [3] \\
$\eta$ & 3.3 & Mamani et al. [36] \\
$\theta$ & $1-\eta(1-\phi)$ & Adida, Dey and Mamani [3] \\
$U$ & Uniform [0,2] & Adida, Dey and Mamani [3] \\
$\mu$ & 1 & Adida, Dey and Mamani [3] \\
$\phi$ & 0.9 & Adida, Dey and Mamani [3] \\
$c$ & {$[0,1.6]$} & Adida, Dey and Mamani [3] \\
$\alpha$ & 0.01 & This paper \\
$\beta$ & 0.015 & This paper \\
$\Delta$ & 0.1 & This paper \\
\hline
\end{tabular}

Note that: (i) The psychological cost of a leftover is 1.53 times greater than the psychological cost of a stockout [8]. Thus, we assume that the psychological cost of overproduction is 1.5 times greater than the psychological cost of underproduction. (ii) In, the production cost c is equal to 0.4 and 1.4, respectively.

\section{References}

1. WHO. World Health Data Platform. Available online: https://www.who.int/data/gho/data/themes/ sustainable-development-goals (accessed on 5 July 2020).

2. Chick, S.E.; Mamani, H.; Simchi-Levi, D. Supply Chain Coordination Influenza Vaccination. Oper. Res. 2008, 56, 1493-1506. [CrossRef]

3. Adida, E.; Dey, D.; Mamani, H. Operational issues and network effects in vaccine markets. Eur. J. Oper. Res. 2013, 231, 414-427. [CrossRef] [PubMed]

4. Yamin, D.; Gavious, A. Incentives' Effect in Influenza Vaccination Policy. Manag. Sci. 2013, 59, $2667-2686$. [CrossRef]

5. Kahneman, D.; Tversky, A. Prospect theory: An analysis of decision under risk. Econometrica 1979, 47, 263-291. [CrossRef]

6. Schweitzer, M.E.; Cachon, G.P. Decision bias in the newsvendor problem with a known demand distribution: Experimental evidence. Manag. Sci. 2000, 46, 404-420. [CrossRef]

7. Koszegi, B.; Rabin, M. A model of reference-dependent preferences. Q. J. Econ. 2006, 121, 1133-1165. 
8. Ho, T.H.; Lim, N.; Cui, T.H. Reference Dependence in Multilocation Newsvendor Models: A Structural Analysis. Manag. Sci. 2010, 56, 1891-1910. [CrossRef]

9. GSK. Responsible Business Supplement 2016. Available online: https://www.gsk.com/media/3610/ responsible-business-supplement-2016.pdf (accessed on 5 July 2020).

10. Deo, S.; Corbett, C.J. Cournot Competition Under Yield Uncertainty: The Case of the US Influenza Vaccine Market. MSom Manuf. Serv. Oper. 2009, 11, 563-576. [CrossRef]

11. Chick, S.E.; Hasija, S.; Nasiry, J. Information Elicitation and Influenza Vaccine Production. Oper. Res. 2017, 65, 75-96. [CrossRef]

12. Mamani, H.; Chick, S.E.; Simchi-Levi, D. A Game-Theoretic Model of International Influenza Vaccination Coordination. Manag. Sci. 2013, 59, 1650-1670. [CrossRef]

13. Dai, T.L.; Cho, S.H.; Zhang, F.Q. Contracting for On-Time Delivery in the US Influenza Vaccine Supply Chain. MSom Manuf. Serv. Oper. 2016, 18, 332-346. [CrossRef]

14. Brito, D.L.; Sheshinski, E.; Intriligator, M.D. Externalities and compulsary vaccinations. J. Public Econ. 1991, 45, 69-90. [CrossRef]

15. Ahlskog, R. When is Blood Thicker Than Water? Variations of Other-Regard in the Vaccination Decision. Available online: http://urn.kb.se/resolve?urn=urn:nbn:se:uu:diva-311018 (accessed on 5 July 2020).

16. Caso, D.; Carfora, V.; Starace, C.; Conner, M. Key Factors Influencing Italian Mothers' Intention to Vaccinate Sons against HPV: The Influence of Trust in Health Authorities, Anticipated Regret and Past Behaviour. Sustainability 2019, 11, 6879. [CrossRef]

17. Guo, F.; Cao, E. Can Reference Points Explain Vaccine Hesitancy? A New Perspective on Their Formation and Updating. Available online: https://doi.org/10.1016/j.omega.2019.102179 (accessed on 5 July 2020).

18. MacDonald, N.E. Vaccine hesitancy: Definition, scope and determinants. Vaccine 2015, 33, 4161-4164. [CrossRef]

19. Su, C.; Liu, X.; Du, W. Green Supply Chain Decisions Considering Consumers' Low-Carbon Awareness under Different Government Subsidies. Sustainability 2020, 12, 2281. [CrossRef]

20. Xu, D.; Long, Y. The Impact of Government Subsidy on Renewable Microgrid Investment Considering Double Externalities. Sustainability 2019, 11, 3168. [CrossRef]

21. Yin, X.; Chen, X.; Xu, X.; Zhang, L. Tax or Subsidy? Optimal Carbon Emission Policy: A Supply Chain Perspective. Sustainability 2020, 12, 1548. [CrossRef]

22. Levi, R.; Perakis, G.; Romero, G. On the Effectiveness of Uniform Subsidies in Increasing Market Consumption. Manag. Sci. 2017, 63, 40-57. [CrossRef]

23. Arifoğlu, K.; Deo, S.; Iravani, S.M.R. Consumption Externality and Yield Uncertainty in the Influenza Vaccine Supply Chain: Interventions in Demand and Supply Sides. Manag. Sci. 2012, 58, 1072-1091. [CrossRef]

24. Ahlskog, R. Democracy and Vaccination Uptake-A Complex Friendship. Available online: http://urn.kb.se/ resolve?urn=urn:nbn:se:uu:diva-311017 (accessed on 5 July 2020).

25. Chemama, J.; Cohen, M.C.; Lobel, R.; Perakis, G. Consumer Subsidies with a Strategic Supplier: Commitment vs. Flexibility. Manag. Sci. 2019, 65, 681-713. [CrossRef]

26. Taylor, T.A.; Xiao, W. Subsidizing the distribution channel: Donor funding to improve the availability of malaria drugs. Manag. Sci. 2014, 60, 2461-2477. [CrossRef]

27. Donohue, K.; Ozer, O.; Zheng, Y.C. Behavioral Operations: Past, Present, and Future. MSom Manuf. Serv. Oper. 2020, 22, 191-202. [CrossRef]

28. Xie, L.; Ma, J.; Goh, M. Supply Chain Coordination in the Presence of Uncertain Yield and Demand. Available online: https://doi.org/10.1080/00207543.2020.1762942 (accessed on 5 July 2020).

29. Giri, B.C.; Bardhan, S.; Maiti, T. Coordinating a three-layer supply chain with uncertain demand and random yield. Int. J. Prod. Res. 2016, 54, 2499-2518. [CrossRef]

30. Cui, T.H.; Raju, J.S.; Zhang, Z.J. Fairness and channel coordination. Manag. Sci. 2007, 53, 1303-1314.

31. Li, M. Overconfident Distribution Channels. Prod. Oper. Manag. 2019, 28, 1347-1365. [CrossRef]

32. Ho, T.H.; Su, X.M.; Wu, Y.Z. Distributional and Peer-Induced Fairness in Supply Chain Contract Design. Prod. Oper. Manag. 2014, 23, 161-175. [CrossRef]

33. Becker-Peth, M.; Thonemann, U.W. Reference points in revenue sharing contracts How to design optimal supply chain contracts. Eur. J. Oper. Res. 2016, 249, 1033-1049. [CrossRef]

34. Xideng, Z.; Bing, X.; Fei, X.; Yu, L. Research on Quality Decisions and Coordination with Reference Effect in Dual-Channel Supply Chain. Sustainability 2020, 12, 2296. [CrossRef] 
35. Yang, D.; Xiao, T. Coordination of a supply chain with loss-averse consumers in service quality. Int. J. Prod. Res. 2017, 55, 3411-3430. [CrossRef]

36. Mamani, H.; Adida, E.; Dey, D. Vaccine market coordination using subsidy. IIE Trans. Healthc. Syst. Eng. 2012, 2, 78-96. [CrossRef]

37. Raz, G.; Ovchinnikov, A. Coordinating Pricing and Supply of Public Interest Goods Using Government Rebates and Subsidies. IEEE Trans. Eng. Manag. 2015, 62, 65-79. [CrossRef]

38. Balakrishnan, A.; Pangburn, M.S.; Stavrulaki, E. "Stack Them High, Let 'em Fly": Lot-Sizing Policies When Inventories Stimulate Demand. Manag. Sci. 2004, 50, 630-644. [CrossRef]

39. Tereyagoglu, N.; Veeraraghavan, S. Selling to Conspicuous Consumers: Pricing, Production, and Sourcing Decisions. Manag. Sci 2012, 58, 2168-2189. [CrossRef]

40. WHO. President BoniYayi Urges African Leaders to Strengthen Health Systems to Contain Epidemics. Available online: http://www.afro.who.int/news/president-boni-yayi-urges-african-leaders-strengthenhealth-systems-contain-epidemics (accessed on 5 July 2020).

41. Arifoglu, K.; Tang, C.S. A Two-Sided Budget-Neutral Incentive Program for Coordinating an Influenza Vaccine Supply Chain with Endogenous Supply and Demand under Uncertainty. Available online: https: //papers.ssrn.com/sol3/papers.cfm?abstract_id=3361140 (accessed on 5 July 2020).

42. Economist, T. So many possibilities, so little time. Economic 2020, 435, 13-16.

43. Bowles, S.; Polania-Reyes, S. Economic Incentives and Social Preferences: Substitutes or Complements? J. Econ. Lit. 2012, 50, 368-425. [CrossRef]

(C) 2020 by the authors. Licensee MDPI, Basel, Switzerland. This article is an open access article distributed under the terms and conditions of the Creative Commons Attribution (CC BY) license (http://creativecommons.org/licenses/by/4.0/). 